\title{
HNF1A deficiency causes reduced calcium levels, accumulation of abnormal insulin granules and uncoupled insulin to C-peptide secretion in a stem cell model of MODY3
}

\section{Bryan Gonzalez ( $\sim$ bryan56@gmail.com )}

Columbia University

Haoquan Zhao

Columbia University

Jacqueline Niu

Columbia University

Damian Williams

Columbia University

Jaeyop Lee

Columbia University

Christopher Goulbourne

Nathan Kline Institute for Psychiatric Research

Yuan Xing

University of Virginia

Yong Wang

University of Virginia

Jose Oberholzer

University of Virginia

Xiajuan Chen

Columbia University

Charles LeDuc

Columbia University

Wendy K Chung

Columbia University

Henry Colecraft

Columbia University Medical Center https://orcid.org/0000-0002-2340-8899

Jesper Gromada

Vertex Pharmaceuticals (United States)

Yufeng Shen

Columbia University https://orcid.org/0000-0002-1299-5979 


\section{Robin Goland}

Columbia University Medical Center

\section{Rudolph Leibel}

Columbia University Medical Center

\section{Dieter Egli}

Columbia University Medical Center https://orcid.org/0000-0002-0812-6412

\section{Article}

Keywords: Maturity Onset Diabetes, HNF1A deficiency, disease

Posted Date: June 1st, 2021

DOl: https://doi.org/10.21203/rs.3.rs-509878/v1

License: (c) (1) This work is licensed under a Creative Commons Attribution 4.0 International License.

Read Full License

Version of Record: A version of this preprint was published at Communications Biology on August 2nd, 2022. See the published version at https://doi.org/10.1038/s42003-022-03696-z. 


\title{
HNF1A deficiency causes reduced calcium levels, accumulation of abnormal insulin granules and uncoupled insulin to $C$-peptide secretion in a stem cell model of MODY3
}

One sentence of summary: Deficiency of the transcription factor $H N F 1 A$ impairs intracellular calcium homeostasis and insulin exocytosis, alters the stoichiometry of insulin to C-peptide release, and leads to an accumulation of abnormal insulin secretory granules in $\beta$-cells.

\begin{abstract}
Mutations in $H N F 1 A$ cause Maturity Onset Diabetes of the Young type 3 (MODY3), the most prevalent form of monogenic diabetes. We generated stem cell-derived pancreatic endocrine cells from human embryonic stem cells (hESCs) with induced hypomorphic mutations in $H N F 1 A$. Using these cells, we show that $H N F 1 A$ orchestrates a transcriptional program required for distinct aspects of $\beta$-cell function. $H N F 1 A$-deficient $\beta$-cells display impaired basal and glucose stimulated-insulin secretion in association with a reduction in $C A C N A 1 A$ and intracellular calcium levels, and impaired insulin granule exocytosis in association with SYT13 down-regulation. Knockout of $P A X 4, C A C N A 1 A$ and $S Y T 13$ reproduce the relevant phenotypes. Reduction of insulin secretion is associated with accumulation of enlarged secretory granules, and altered stoichiometry of secreted insulin to C-peptide. In HNF1A deficient $\beta$-cells, glibenclamide, a sulfonylurea drug used in the treatment of MODY3 patients, increases intracellular calcium, and thereby restores Cpeptide and insulin secretion to a normal stoichiometric ratio. To study $H N F 1 A$ deficiency in the context of a human disease model, we also generated stem cell-derived pancreatic endocrine cells from two MODY3 patient's induced pluripotent stem cells (iPSCs). While insulin secretion defects are constitutive in cells with complete $H N F 1 A$ loss of function, $\beta$-cells heterozygous for
\end{abstract}


hypomorphic $H N F 1 A$ mutations are initially normal, but lose the ability to secrete insulin and acquire abnormal stoichiometric secretion ratios, while gene corrected cells remain normal. Our studies provide the molecular basis for the treatment of MODY3 with sulfonylureas, and demonstrate promise for the use of cell therapies for MODY3.

\section{Introduction}

Maturity onset diabetes of the young (MODY) is an autosomal dominant form of diabetes with onset typically before the age of 25 years accounting for $1-2 \%$ of diabetes incidence (1). There are at least 11 (2) genetically distinct types of MODY, due to derangements in $\beta$-cell development or function. MODY3 is caused by mutations in the transcription factor $\operatorname{HNF} 1 \mathrm{~A}(3,4)$ and accounts for $63 \%$ of diagnosed instances of MODY (5). MODY3 patients have normal glucose tolerance during childhood and early adult life, but show progressive reduction of insulin secretion in response to glucose (6-9). Glycemia typically increases over time, resulting in the need for treatment with the insulin secretory sulfonylurea drugs. Eventually, 30-40\% of patients become insulin-dependent due to progressive deterioration of $\beta$-cell function.

HNF1A is a 631-amino acid transcription factor with three major domains: dimerization, DNAbinding, and transactivation. Over $200 H N F 1 A$ diabetes-related mutations have been identified in all major ethnic groups (10) and the DNA-binding domain has the highest frequency of mutation in MODY3 patients (5). Understanding the role of $H N F 1 A$ gene and the pathophysiology of MODY3 has been difficult because of the difficulty in studying human islets or mass with these mutations. Mouse models of HNF1A deficiency do not accurately mimic patient phenotypes (11). 
Stem cell-derived $\beta$-cells provide a useful model system, and have been used to study $\beta$-cell development in humans $(12,13)$ and to recapitulate disease phenotypes $(14,15)$. Differentiation of pluripotent stem cells to pancreatic endocrine cells can be achieved by a multistep protocol resulting in islet-like clusters containing all endocrine cell types (16-18). Transplanting these isletlike clusters into mice allows functional testing of stem cell-derived $\beta$-cells (sc $\beta$-cells) in vivo (19, 20). Here we show that human stem cell-based models of HNF1A deficiency shows islet developmental bias towards $\alpha$-cells, altered insulin granule morphology and the stoichiometry of insulin:C-peptide secretion in vitro and in vivo. We used these models to identify disordered calcium homeostasis and glucose-mediated exocytosis as key mechanisms accounting for the secretory defects observed. This study was designed using two cell-based models: Human embryonic stem cell (hESC) lines null for $H N F 1 A$ in Fig. 1-5 and Fig. S1-S10; and induced pluripotent stem cell (iPSC) lines with MODY3 patient-specific mutations in Fig. 6 and Fig. S11S13. Fig. S1 provides a schematic overview of the study.

\section{Results}

\section{Generation of isogenic cell lines with $H N F 1 A$ mutations in hESCs and MODY3 iPSCs}

To elucidate the cellular functions of $H N F 1 A$, we used CRISPR/Cas9 technology to generate hESC lines (Mel1) harboring non-naturally occurring heterozygous and homozygous null mutations. The Mel1 hESC line has a $I N S^{G F P / w t}$ and $G A P D H^{\text {Luciferase/wt }}$ dual-reporter containing a GFP gene knockin in place of a coding INS locus, and a luciferase knockin in one GAPDH locus (21). GFP expression under the control of the insulin promoter does not alter $\beta$-cell function in mice (22) and does not cause ER-stress in human sc $\beta$-like-cells (23). This marker enables imaging and isolation of viable INS-GFP ${ }^{+}$cells in vitro and in vivo. We designed short guide RNAs (sgRNAs) to 
introduce indel mutations in the DNA-binding domain (exon 3) of the $H N F 1 A$ gene (Fig. S2A and Table S1) because the DNA-binding domain has the highest frequency of mutation in MODY3 patients (5). Transfection of hESC lines with Cas9-GFP and sgRNAs \#12 or \#14, followed by sorting for GFP (Fig. S2B), achieved 17.3 and 20.4\% indel efficiency for the sgRNAs as shown by surveyor assay (Fig. S2C). Gene editing was efficient, resulting in compound heterozygous knockouts and heterozygous mutations (Fig. S2D) with no off-target mutations detected through targeted sequencing (Table S2). Heterozygous (hESC HNF1A Het) and compound heterozygousnull mutant cell lines (hESC HNF1A KO1 and KO2) with different indels in exon 3 and premature protein termination were chosen for further studies (Fig. S2E). hESC HNF1A Het, KO1 and KO2 lines retained the HNF1A dimerization domain (truncated HNF1A), while hESC HNF1A KO3 line was mutated at the start codon, deleting the entire HNF1A protein (Fig. S2E).

To understand the consequences of MODY3 patient-specific heterozygous mutations, we also generated iPSC lines from MODY3 patient fibroblasts containing heterozygous mutations in the transactivation domain (MODY3 iPSC Het: +/460_461insCGGCATCCAGCACCTGC); and DNA-binding domain (MODY3 iPSC Het: +/R200Q). The R200Q variant has been previously associated with MODY3 (24); however, the precise effect of this missense mutation on $H N F 1 A$ function is unknown, but very likely pathogenic (24). Using CRISPR/Cas9, we corrected the R200Q mutation from MODY3 iPSC Het (Fig. S2F) with an efficiency of $7.9 \%$ (5 clones out of $63)$ to generate isogenic wildtype cells. In addition to the hESC knockout lines, we also generated iPSCs compound heterozygous knockout lines with an efficiency of 42.9\% (27 clones out of 63) (Fig. S2D). An iPSC line with a deletion of the WT allele, but intact patient's mutation (MODY3 iPSC: R200Q/-) was chosen for further studies (Fig. S2G). We also introduced the same R200Q 
mutation into both alleles in the hESC line (hESC Hom: R200Q/R200Q) (Fig. S2H) with an efficiency of 20.8\% (5 clones out of 24) (Fig. S2I). All genetically manipulated cell lines (Fig. S2J) resulted in modified versions of HNF1A protein (Fig. S2K and S2L) and had a normal karyotype (Fig. S2M and S2N) and no off-target mutations (Table S2).

\section{HNF1A is not required to generate pancreatic endocrine cells}

To determine the functional consequences of $H N F 1 A$ deficiency, the first part of this study will focus on the hESC model. Stem cells were differentiated into the pancreatic lineage using a published differentiation protocol (20) (Fig. S3A). Differentiation of wildtype stem cells using this protocol consistently generated $82 \% \mathrm{PDX1}^{+} / \mathrm{NKX} 6.1^{+}$cells at the pancreatic progenitor stage (day 11). At the endocrine stage (day 27 ), we obtained $45 \% \mathrm{PDX}^{+} / \mathrm{CPEP}^{+}$cells with $60 \%$ of them coexpressing NKX6.1 (sc $\beta$-like cells), $30 \% \mathrm{GCG}^{+}$cells ( $\operatorname{sc} \alpha$-like cells) and $10 \% \mathrm{SST}^{+}$cells $(\mathrm{sc} \delta$ like cells) (Fig. S3B).

To determine the timing of HNFIA expression, we performed qPCR at different stages of differentiation. Insulin mRNA was detected at the endocrine stage (day 20) (Fig. S3C), whereas HNF1A mRNA is first detected at low levels at the primitive gut tube stage (day 5), increased at the pancreatic progenitor stage (day 11) and increased further at the endocrine stage (day 27) (Fig. S3D), identifying the stages where lack of $H N F 1 A$ could have developmental and/or functional consequences. $H N F 1 A$ knockout resulted in significant reduction of $H N F 1 A$ transcript at the endocrine stage (Fig. S3E); HNF1A protein was detected in hESC WT, but only a faint band was detected in hESC HNF1A KO-derived endocrine cells by western blot (Fig. S3F). 
Mutations in $H N F 1 A$ did not affect hESC lines in generating definitive endoderm cells (SOX17 ${ }^{+}$) at day 3 of differentiation or pancreatic progenitor cells $\left(\mathrm{PDX}^{+} / \mathrm{NKX} 6.1^{+}\right)$at day 11 of differentiation as determined by immunohistochemistry (Fig. S3G). At the endocrine stage of development (day 27), cell clusters or organoids were morphologically indistinguishable. hESC lines became positive for INS-GFP fluorescence due to the $I N S^{G F P / w t}$ reporter; there was no differences in GFP expression by genotype (Fig. S3H). HNF1A KO cells were capable of differentiation to all islet endocrine cell types, including $\mathrm{PDX}^{+} / \mathrm{NKX} 6.1^{+} / \mathrm{SYP}^{+} / \mathrm{CPEP}^{+}$cells $(\operatorname{sc} \beta$ like cells), $\mathrm{GCG}^{+}$cells (sc $\alpha$-like cells) and $\mathrm{SST}^{+}$cells ( $\mathrm{sc} \delta$-like cells) (Fig. 1A and S3I-S3K) indicating that $H N F 1 A$ is not required to generate pancreatic endocrine cells. No differences were found for PDX1 and NKX6.1 in hESC HNF1A WT and HNF1A KO endocrine cells (day 27) by immunohistochemistry (Fig. S3J and S3K) and flow cytometry (Fig. S3L). The ability of $H N F 1 A$ KO cells to differentiate to all endocrine cell types enabled the comparison of transcriptional programs in $H N F 1 A \mathrm{KO}$ and WT cells.

HNF1A deficiency impairs a network of genes required for calcium signaling, glucosestimulated insulin exocytosis and $\beta$-cell fate

To determine the transcriptional consequences of $H N F 1 A$ deficiency in sc $\beta$-cells, bulk and singlecell RNA sequencing was performed in INS-GFP sorted $\beta$-like cells derived from hESC HNF1A $\mathrm{KO}$ and WT lines in vitro. Volcano plot analysis of bulk RNA sequencing identified 30 upregulated genes and 148 down-regulated genes, including $H N F 1 A$ in hESC $H N F 1 A$ KO $\beta$-like cells (Fig. 1B and Table S3). Single-cell RNA sequencing showed that hESC HNF1A KO $\beta$-like cells segregated from hESC HNF1A WT $\beta$-like cells based on their gene expression profile by principal component analysis (Fig. 1C), and volcano plot analysis (Fig. 1D) revealed previously 
undescribed HNF1A target genes involved in intracellular calcium signaling (CACNA1A, S100A6 and TMEM37), calcium-mediated insulin secretion (KCNH6) and glucose-stimulated insulin secretion $(R F X 6, D P E P 1, D P P 4$ and $I A P P)$ (Fig. 1E) as commonly downregulated with bulk RNA sequencing from $H N F 1 A \mathrm{KO}$ sc $\beta$-like cells. Among the up-regulated genes in hESC $H N F 1 A \mathrm{KO}$ $\beta$-like cells, we found genes involved in synaptic vesicle cycle (AP2A2, SH3KBPI and HSPA1B) (Fig. 1E and Table S4).

To understand the transcriptional consequences of HNF1A mutation in other endocrine cell types, we analyzed all single cells within the islet-like clusters, allowing the segregation of cell types according to single-cell gene expression. In addition to $H N F 1 A \mathrm{KO}$ lines, an $\mathrm{hESC}$ line homozygous for HNF1A R200Q (R200Q/R200Q), a MODY3 patient-specific mutation, was included. Using clustering analysis from those cells, we grouped the cells into thirteen different cell type populations (Fig. S4A and S4B). To cluster different endocrine cells, we used the expression of endocrine cell markers such as SYP, INS, GCG and SST (Fig. S4C) and pancreatic progenitor markers $N K X 6.1, P D X 1, M A F A$ and $H N F 1 A$ (Fig. S4D) from all cell lines. This allowed the identification of insulin expressing cells (cluster 0) and glucagon expressing cells (cluster 2) (Fig. 1F). From the population of insulin expressing cells (cluster 0), cells defined by high insulin and low glucagon expression were considered sc $\beta$-like cells and were compared between genotypes (Fig. S5A).

Separation into populations according to these markers enabled us to identify differentially expressed genes in $H N F 1 A$ mutant cells affecting specific endocrine cell types. In sc $\beta$-like cells, a total of 73 genes were differentially expressed between $H N F 1 A$ WT, homozygous R200Q and KO 
lines (Fig. 1G and Table S5). Pathway analysis of differentially expressed genes in sc $\beta$-like cells revealed genes involved in MODY, endocrine cell development, calcium signaling/sensing, insulin secretion and synaptic vesicle cycle (Fig. S5B). Furthermore, several MODY genes (RFX6, PAX4 and NEUROD1) were down-regulated (Fig. 1G). These transcription factors are known to be important for the functional identity of adult pancreatic $\beta$-cells $(25,26)$. We also identified down-regulated genes important for intracellular calcium signaling (S100A6 and TMEM37), exocytosis-regulated insulin secretion (SYT13) and glucose-stimulated insulin secretion (IGF2 and LLGL2) in hESC HNF 1A mutated $\beta$-like cells (Fig. 1I and Table S5) $(25,27,28)$. Among the upregulated genes, we found genes involved in synaptic vesicle cycle $(R A B 3 A$ and $V A M P 2)$ (Fig. 1G and Table S5). RFX6, KCNH6, S100A6, AICF, DPEP1 and DPP4 were the most consistently downregulated genes in hESC HNF1A mutated $\beta$-like cells. In addition to those genes, SYT13, $C A C N A 1 A$ and $P A X 4$ were down-regulated in the sc $\beta$-like-cell subpopulation (Fig. 1H). Similar to our study, a recent case study of cadaveric human islets of a MODY3 patient (+/T260M) also found down-regulation of $R F X 6, C A C N A 1 H$, IAPP and TMEM37 in $\beta$-cells, and down-regulation of CACNA1A, RFX6, PCBD1 and PPP1R1A in $\alpha$-cells (29). These genes are required for calcium signaling, glucose-stimulated insulin exocytosis, and endocrine cell fate.

To determine the molecular consequences of $H N F 1 A$ deficiency in other endocrine cell types, we analyzed mono-hormonal sc $\alpha$-like cells (cluster 2a), characterized by high glucagon and low insulin expression (Fig. S5C and S5D). We identified up-regulated genes involved in glucagon signaling pathways (PKM, CALM1 and CALM2) and down-regulated genes involved in insulin secretion (ADCY1, KCNH6, RFX6,IGF2, TM4SF4 and ATF4), calcium-mediated exocytosis (SYT7) and $\beta$-cell dedifferentiation $(G C)$ (30) (Fig. S5E and S5F). Furthermore, in bi-hormonal 
cells expressing both insulin and glucagon, $P Y Y$ was down-regulated, while $G C G$ was up-regulated (Fig. S5G and S5H) in $H N F 1 A$ mutated lines, indicating a transcriptional bias of endocrine cells towards the $\alpha$-cell fate.

A recent publication by Cardenas-Diaz et al. (31) identifies LINC01139 as an HNF1A target implicated in $\beta$-cell dedifferentiation and $\beta$-cell respiration. However, we found no significant difference in the expression of this long non-coding RNA between WT and $H N F 1 A$ mutated (hESC Het, hESC Hom R200Q/R200Q, hESC KO or iPSC MODY3 Het +/460ins) $\beta$-like (Fig. S5I-5K) and $\alpha$-like cells (hESC Hom R200Q/R200Q and hESC KO) (Fig. S5L). Similar results were reported in cadaveric human $\beta$-cells from non-diabetic donors as compared to one MODY3 donor (+/T260M) (Fig. S5M).

In summary, $H N F 1 A$ orchestrates a network of genes promoting endocrine differentiation to $\beta$ cells, and in differentiated $\beta$-cells regulates genes involved in calcium signaling, hormone exocytosis and glucose-stimulated insulin secretion.

\section{HNF1A deficiency causes a developmental bias towards the $\alpha$-cell fate}

Downregulation of genes involved in adult $\beta$-cell identity ( $P A X 4$ and $R F X 6)$ with transcriptional bias towards the $\alpha$-cell fate in $H N F 1 A$ KO cells point to a developmental role of HNF1A. Though both hESC HNF1A KO and WT cells gave rise to similar numbers of insulin positive cells (Fig. S6A and S6B), immunostaining showed a variable increase in sc $\alpha$-like cell numbers from $30 \%$ to up to $70 \%$ in the hESC HNF1A KO line (Fig. 2A and S6B). This was confirmed by a significant increase in $\mathrm{GCG}^{+} / \mathrm{CPEP}^{+}$cell type ratio by immunohistochemistry (Fig. 2B) and glucagon content 
by ELISA (Fig. 2C). Furthermore, quantification by flow cytometry consistently showed a significant increase in sc $\alpha$-like cells in three different hESC HNF1A KO lines as compared to hESC HNF1A WT lines (Fig. S6C and S6D). The absolute increase in sc $\alpha$-like cells in hESC $H N F 1 A \mathrm{KO}$ cells was driven by an increase in the number of bi-hormonal ( $56 \%$ in $\mathrm{KO}$ vs. $20 \%$ in WT) and mono-hormonal (7\% vs. 1\%) sc $\alpha$-like cells as shown by flow cytometry (Fig. 2D), immunohistochemistry (Fig. S6E), and RNA sequencing (Fig. S6F). Transcriptional analysis by single-cell RNA sequencing of glucagon and insulin double hormone-positive cells showed an increase in glucagon gene expression and a decrease in insulin gene expression in hESC HNF1A KO cells compared to WT cells (Fig. 2E). These findings are consistent with PAX4 repressing pancreatic glucagon gene expression (32), and indicate that HNF1A hypomorphism affects endocrine cell fate by increasing expression of the glucagon gene in pancreatic endocrine cells. $H N F 1 A$ deficiency did not affect the proportion of sc $\delta$-like cell fate as shown by flow cytometry (Fig. S6G and S6H). No effects of HNF1A mutations on proliferation (Fig. S6I) or survival (Fig. S6J and S6K) of sc $\beta$-like cells were observed by flow cytometry. These results show that HNF1A deficiency biases endocrine cells toward the $\alpha$-cell fate in vitro.

In order to assess the developmental requirements of $H N F 1 A$ in endocrine cells in vivo, we transplanted pancreatic islet-like clusters derived from hESC HNF1A WT and hESC HNF1A KO lines with $G A P D H^{L u c i f e r a s e / w t}$ and $I N S^{G F P / w t}$ dual-reporter (21). Mice received $\sim 180$ clusters of stem cell-derived islet-like cells (Fig. S7A) containing similar amount of CPEP/PDX1 positive cells across genotypes (Fig. S7B and S7C); but with different amounts of bihormonal GCG/CPEP positive cells (Fig. 2D). Cell Transplantation was done by injection into the ventral and medial muscle groups of the left quadriceps in NOD SCID gamma immunodeficient mice (NSG mice) 
(Fig. 2F). The skeletal muscle was chosen for its dense vasculature and easy access for surgical procedures. Skeletal muscle has been used for other endocrine transplants, including for parathyroid auto-transplantation in patients undergoing parathyroidectomy with a $93 \%$ success rate (33). Analysis of engraftment efficiency was evaluated by bioluminescence intensity (BLI) several weeks post-transplantation. Mice with successful engraftment showed a two-fold increase in BLI 4 to 6 weeks post transplantation, while those with failed engraftment showed a decrease (Fig. S7D). Transplantation was successful in 79\% (31/39) of mice, independent of HNF1A genotype (Fig. S7E). 92.5\% (49/53) of mice transplanted with hESC lines remained teratoma-free (Fig. S7F). In those mice, the graft explant (30 weeks post-transplantation) (Fig. S7G) was a vascularized tissue of about $220 \mathrm{mg}$ (Fig. S7H). According to luminescence intensity, there was a gradual increase in BLI from week 0 to 30 post-transplantation without significant differences by $H N F 1 A$ genotype (Fig. 2G). Grafts remained localized for up to 50 weeks post-transplantation, and in no case (0/39) was luminescence detected in an ectopic location. These results show that skeletal muscle is a stable and suitable transplantation site for SC-derived islet-like cells.

Thirty weeks post-transplantation, we isolated grafts from normoglycemic animals. Quantification of sc $\beta$-mass as determined by GFP fluorescence did not differ by HNF1A genotype (Fig. 2H-2I and S7I-S7J). The presence of exclusively mono-hormonal endocrine cells, including sc $\beta$-cells $\left(\mathrm{CPEP}^{+} / \mathrm{PDX}^{+} / \mathrm{NKX} 6.1^{+}\right)$, as well as glucagon- and somatostatin-positive cells were apparent in both hESC HNF1A WT and hESC HNF1A KO grafts (Fig. 2J). No double hormone-positive cells were identified in cells of either genotype. Consistent with the in vitro studies, the percentage of sc $\alpha$-cells in $H N F 1 A$ KO compared to $H N F 1 A$ WT islet-like structures were increased by $24 \%$ (Fig. $\mathbf{2 K}$ and $\mathbf{S 7 K}$ ), leading to a significant increase in $\mathrm{GCG}^{+} / \mathrm{CPEP}^{+}$cell type ratio (Fig. $2 \mathrm{~L}$ ); similar 
results as found in cadaveric human islets of a MODY3 donor (+/T260M) (29) (Fig. 2K). Consistent with this observation, ex vivo analysis of isolated hESC HNF1A KO grafts showed an increase in glucagon protein content by ELISA (Fig. 2M and S7L-S7M). Glucagon secretion was higher in hESC HNF1A KO grafts when stimulated with glibenclamide, a second-generation sulfonylurea drug compared to hESC HNF1A WT grafts (Fig. 2N). Similarly, elevated glucagon secretion upon $\mathrm{KCl}$ stimulation was detected in vitro from hESC $H N F 1 A \mathrm{KO}$-derived endocrine cells (Fig. S7N). These results point not only to a gain of $\alpha$-cell number, but also enhanced $\alpha$-cell function due to $H N F 1 A$ deficiency. This is consistent with the up-regulation of genes (PKM, CALM1 and CALM2) involved in glucagon signaling pathways in sco-like cells.

To determine whether the downregulation of PAX4 seen in HNF1A mutant cells (Fig. 1H) could reproduce the bias towards the $\alpha$-cells, we generated hESC PAX4 KO cell lines using CRISPR/Cas9 (Fig. S7O). The PAX4 gene is an essential gene for differentiation of insulinproducing $\beta$-cells in the mammalian pancreas (34). Knockout of $P A X 4$ in rabbits induces a decreased in number of $\beta$-cells and increased number of $\alpha$-cells (26), whereas overexpression of $P A X 4$ reduces glucagon expression in differentiating hESCs (35). Endocrine cells differentiated from an hESC PAX4 KO line were characterized by significant decrease in sc $\beta$-like cells compared to hESC WT and HNF $1 A$ KO lines. In the hESC PAX4 KO lines, sc $\alpha$-like cells were increased to levels similar to hESC HNF 1A KO cells by immunohistochemistry (Fig. 20) and flow cytometry (Fig. S7P). Therefore, in $H N F 1 A \mathrm{KO}$ lines, down-regulation of $P A X 4$ and de-repression of glucagon gene are associated with the developmental bias towards $\alpha$-cell fate. 
In summary, $H N F 1 A$ is not required for development of mono-hormonal endocrine cells ( $\alpha-$, $\beta$ and $\delta$-cells). But restriction of HNF1A activity increases the number and proportion of $\alpha$-cells in association with $P A X 4$ downregulation. As $H N F 1 A$ clearly regulates a network of genes, a role of additional $H N F 1 A$ target genes in $\beta$-and $\alpha$-cell fate is likely.

\section{$H N F 1 A$ deficiency affects glucose-mediated exocytosis of insulin granules}

To determine the consequences of $H N F 1 A$ deficiency on $\beta$-cell function, we measured glucosestimulated insulin secretion. hESC $H N F 1 A \mathrm{KO}$ sc $\beta$-like cells had reduced basal insulin secretion (Fig. S8A) and impaired glucose-stimulated insulin secretion compared to WT sc $\beta$-like cells and compared to human pancreatic islets in vitro (Fig. 3A). These differences in hormone secretion between $H N F 1 A$ mutant and $H N F 1 A$ WT sc $\beta$-like cells were not due to a reduction in insulin content (Fig. S8B). In contrast to glucose, treatment with the insulin secretagogues, tolbutamide or potassium chloride resulted in insulin secretion in hESC HNF1A KO cells comparable to hESC HNF1A WT $\beta$-like cells (Fig. 3B). Thus, membrane depolarization enables recruitment of an insulin granule reservoir that is abnormally retained in $H N F 1 A$ mutant $\beta$-cells and fails to be secreted in response to glucose. The ability of sulfonylureas in releasing insulin from $\beta$-cell granules is consistent with the clinical efficacy of sulfonylurea drugs in MODY3. These defects are likely intrinsic to the $\beta$-cell, and not due to paracrine effects of glucagon (36).

As calcium signaling is a mechanism for the release of insulin through granule exocytosis, we measured intracellular calcium levels in dispersed sc $\beta$-like cells in vitro. Both genotypes had glucose-stimulated calcium responses (Fig. 3C). However, hESC HNF1A KO $\beta$-like cells had significantly lower calcium levels relative to hESC HNF1A WT sc $\beta$-like cells as measured by 
Fura-2 fluorescence (Fig. 3C). This was further supported by measurements of absolute intracellular calcium concentrations by Fura-2 fluorescence (Fig. 3D). Among the HNF1A target genes, reduced expression of $C A C N A 1 A$ is potentially involved in reducing intracellular calcium levels, and SYT13 may be required for efficient exocytosis-mediated insulin secretion. CACNA1A encodes a voltage-dependent calcium channel mediating the entry of calcium into excitable cells and is involved in calcium-dependent insulin secretion and type 2 diabetes (37). Synaptotagmins are calcium sensors localized in the $\beta$-cell insulin granules, and are required for vesicle fusion and glucose-stimulated insulin release (38-40). SYT13 is a member of the synaptotagmin family and is predicted to be involved in calcium-regulated exocytosis. SYT13 is down-regulated in human T2D islets and silencing of SYT13 impairs insulin secretion in INS1-832/13 cells (41). To determine whether down-regulation of $C A C N A 1 A$ and $S Y T 13$ seen in $H N F 1 A$ mutant cells (Fig. 1H) reproduced the reduced intracellular calcium levels and reduced glucose-stimulated insulin secretion, we generated hESC CACNA1A KO and SYT13 KO cell lines using CRISPR/Cas9. Sanger sequencing revealed homozygous mutations resulting in frame shifts (Fig. S8C). Differentiation of hESC CACNA1A KO and hESC SYT13 KO generated INS-GFP organoids (Fig. 3E) comprised of $\sim 51 \%$ PDX1/CPEP and $~ 16 \%$ GCG positive cells (Fig. 3F and S8D) with no differences compared to isogenic WT cells. Dispersed hESC CACNA1A KO $\beta$-like cells (Fig. S8E) had significantly reduced intracellular calcium levels compared to hESC WT cells (Fig. 3G and S8F). The reduction of intracellular calcium in hESC CACNA1A $\mathrm{KO}$ was intermediate to hESC $H N F 1 A \mathrm{KO}$ cells, indicating that $C A C N A 1 A$ is not the only gene affecting intracellular calcium levels in hESC HNF1A KO cells. Intracellular calcium levels in SYT13 KO where unchanged compared to WT cells (Fig. 3G and S8F). Reduced basal and stimulated insulin secretion were observed in both hESC CACNA1A KO and hESC SYT13 KO sc $\beta$-like cells at intermediate levels 
to $H N F 1 A \mathrm{KO}$ sc $\beta$-like cells (Fig. 3H). Treatment with tolbutamide or $\mathrm{KCl}$ stimulated insulin secretion in all KO lines comparable to the corresponding WT lines. Impairments in insulin release due to reduced levels of these molecules can be overcome by elevating calcium beyond physiological levels using tolbutamide or $\mathrm{KCl}$. The milder phenotypes of cells deficient for single target genes compared to $H N F 1 A$ KO cells suggests that the combined effects on several HNF1A target genes may be responsible for reduced glucose-stimulated insulin secretion.

\section{HNF1A deficiency alters the stoichiometry of insulin to C-peptide secretion}

To interrogate the function of hESC HNF1A KO $\beta$-cells in vivo, we monitored circulating human insulin and C-peptide concentrations in euglycemic mice transplanted with sc-islet-like clusters. By 4 weeks post-transplantation, plasma human C-peptide concentrations were significantly lower in mice transplanted with hESC HNF1A KO $\beta$-cells compared to hESC HNF1A WT $\beta$-cells (Fig. 4A). Circulating human C-peptide concentrations in mice transplanted with hESC HNF1A WT $\beta$ cells increased over time, reaching human physiological levels (652 \pm 146 pM) 24-30 weeks posttransplantation. In mice transplanted with hESC $H N F 1 A \mathrm{KO} \beta$-cells, plasma human C-peptide concentrations increased to a maximum of $352 \pm 142 \mathrm{pM}$ at 30 weeks post-transplantation (Fig. 4A). In mice transplanted with hESC HNF1A WT $\beta$-cells, human insulin was detected as early as 4 weeks post-transplantation and concentrations increased over time, reaching human physiological levels $(12.74 \pm 2.1 \mathrm{mU} / \mathrm{L})$ 24-30 weeks post-transplantation. However, mice transplanted with hESC $H N F 1 A \mathrm{KO} \beta$-cells had virtually undetectable plasma human insulin concentration for 30 weeks (Fig. 4B) despite high plasma human C-peptide levels (>300 pM). These differences in hormone secretion between $H N F 1 A$ mutant and $H N F 1 A$ WT sc $\beta$-cells were not due to reduced sc $\beta$-cell mass in vivo (Fig. 2I). These findings in vivo led us to quantify the 
secretion ratio of those hormones in sc $\beta$-cells in vitro. For equivalent secretion of human C-peptide at basal glucose condition (Fig. S8G), secretion of human insulin was significantly reduced in hESC HNF1A KO lines (Fig. S8H), leading to decreased insulin:C-peptide secretion ratios in vitro (Fig. S8I). Thus, the absence of insulin in the plasma was not a limitation of the sensitivity of the assay but a defect in the stoichiometry of insulin to C-peptide in circulation. While plasma of mice grafted with hESC HNF1A WT $\beta$-cells consistently showed an insulin:C-peptide molar ratio of $0.22 \pm 0.11$ from week 4 to 30 post-transplantation, mice grafted with hESC $H N F 1 A$ KO $\beta$-cells had an 18-fold lower ratio (Fig. 4C). This decrease in circulating insulin:C-peptide ratio was reciprocal to a 3-fold increase in the insulin:C-peptide ratio of intracellular content from hESC $H N F 1 A \mathrm{KO}$ isolated grafts (Fig. 4D), demonstrating complementary imbalance of insulin to C-peptide. The altered insulin:C-peptide ratio was not due to differences in insulin processing since insulin:proinsulin ratios in hESC $H N F 1 A \mathrm{KO}$ and hESC $H N F 1 A$ WT grafts were identical in vivo (Fig. 4E) and in vitro (Fig. S8J-S8L) and no differences were found in the transcript levels of processing genes $(P C 1 / P C 3)$ in RNA sequencing analysis (Table S3-S5). Impaired stoichiometry of circulating insulin to C-peptide was also observed in mice transplanted with two additional homozygous mutant hESC lines (Fig. S8M). Therefore, HNF1A deficiency not only impairs insulin secretion, but also the stoichiometry of circulating insulin to C-peptide ratios.

To evaluate glucose-stimulated insulin secretion, we performed an intraperitoneal glucose tolerance test (iPGTT) in transplanted mice. During fasting and following an iPGTT, mice transplanted with hESC HNF1A WT $\beta$-cells displayed normal human insulin and C-peptide secretion profiles (Fig. 4F-4H). In contrast, hESC $H N F 1 A$ KO $\beta$-cells were non-responsive to glucose: plasma human C-peptide was not decreased by fasting and did not increase after glucose 
injection with concentrations significantly lower than in mice transplanted with hESC HNF1A WT $\beta$-cells (Fig. 4G). Remarkably, circulating human insulin remained undetectable in animals engrafted with hESC HNF1A KO $\beta$-cells despite high plasma levels of human C-peptide (Fig. 4H). Therefore, HNF1A deficiency affects glucose-stimulated insulin secretion, and C-peptide is released in a constitutive manner independent of metabolic state, resulting in an altered ratio of insulin to C-peptide.

\section{Glibenclamide restores insulin secretion in $H N F 1 A$ deficient sc $\beta$-cells}

In contrast to glucose, intra-peritoneal injection of the sulfonylurea drug, glibenclamide, in mice transplanted with hESC $H N F 1 A$ KO $\beta$-cells showed a significant increase in C-peptide 10 minutes after glibenclamide injection (Fig. 4I). Surprisingly, human insulin concentrations in mice transplanted with hESC $H N F 1 A \mathrm{KO} \beta$-cells increased from undetectable levels to $14 \pm 21 \mathrm{mU} / \mathrm{L}$, reaching levels comparable to those in the control group (Fig. 4J). Insulin:C-peptide ratios in plasma of animals transplanted with hESC HNF1A KO $\beta$-cells were increased 10-fold by glibenclamide, equal to the ratios in mice transplanted with hESC HNF1A WT $\beta$-cells (Fig. 4K).

Clearance of insulin from the circulation occurred with the same kinetics in mice grafted with hESC HNF1A WT and hESC HNF1A KO $\beta$-cells 20 minutes after glibenclamide administration (Fig. 4K), excluding insulin stability or clearance in plasma as the cause for the low insulin levels in mice transplanted with $H N F 1 A$ mutant sc $\beta$-cells. To further test whether the reduced insulin:Cpeptide secretion ratios from hESC HNF1A KO $\beta$-cells were due to secretory defects and not insulin stability or clearance, we isolated the hESC $H N F 1 A \mathrm{KO}$ grafts from mice and identified a significant decrease in insulin:C-peptide secretion ratios compared to HNF1A WT grafts ex vivo. This ratio was restored after exposure of the grafts to glibenclamide (Fig. 4L). 
Thus, membrane depolarization initiated by closure of the ATP-sensitive $\mathrm{K}^{+}\left(\mathrm{K}_{\mathrm{ATP}}\right)$ channels (target of glibenclamide) and high intracellular calcium levels allow the secretion of insulin granules that are abnormally retained in $H N F 1 A$ mutant $\beta$-cells.

\section{HNF1A deficiency causes abnormal insulin granule structure}

To identify the basis of the insulin secretory defect in hESC HNF1A KO $\beta$-cells we examined insulin granule morphology by electron microscopy from 30-week explants of normoglycemic mice. hESC HNF1A WT grafts were comprised of sc $\beta$-cells with a characteristic high-electron density core separated from limiting membranes by a "halo" (Fig. 5A and S9A). These morphologies are consistent with electron microscopy of normal human $\beta$-cells. In contrast, hESC $H N F 1 A$ KO islets-like were comprised of sc $\beta$-cells where the majority $(>90 \%)$ of the insulin granules were abnormally enlarged (Fig. 5A-5C) with a diffuse electron-light insulin core (Fig. 5D-5E and S9A), characteristic of immature secretory granules. Enlarged insulin granules were further confirmed by immunogold staining for human C-peptide (Fig. 5F). Similar results were observed in vitro, where hESC HNF1A mutated cells had increased insulin granule diameter with a diffuse light insulin core (Fig. S9B and S9C). For sc $\alpha$-cells, no differences in morphology, structure or granule size between $H N F 1 A$ genotypes was seen (Fig. S9D). While these abnormal insulin granules are not secreted in response to glucose, they can be secreted in response to sulfonylureas. These results show that $H N F 1 A$ is required for normal insulin granule formation and function.

HNF1A deficient sc $\beta$-cells are unable to maintain glucose homeostasis in diabetic mice 
To test the ability of hESC HNF1A KO $\beta$-cells to maintain in vivo normoglycemia, endogenous murine $\beta$-cells were ablated by the administration of streptozotocin (STZ) 22 weeks posttransplantation. STZ is a drug that, by virtue of species-related difference in glucose transporters, specifically targets murine $\beta$-cells without affecting human $\beta$-cells (42). We first confirmed that HNF1A deficiency did not increase sensitivity of human cells to STZ. There was no change in cell death (Fig. S10A) or C-peptide secretion (Fig. S10B) when HNF1A WT or KO sc $\beta$-like-cells were exposed to STZ in vitro. In vivo, circulating plasma human C-peptide from mice transplanted with $H N F 1 A$ WT or $H N F 1 A$ KO cells was unchanged or increased post STZ treatment (Fig. 5G). Mouse C-peptide was undetectable by ELISA (Fig. S10C) and immunohistochemistry of the pancreas (Fig. S10D) demonstrating successful and specific ablation of mouse $\beta$-cells. Mice transplanted with the hESC HNF1A WT $\beta$-cells were normoglycemic $(\sim 100 \mathrm{mg} / \mathrm{dl}$ and $\mathrm{HbA} 1 \mathrm{C} \sim 5 \%)$ for at least 10 weeks post STZ injection (Fig. 5H and S10E) and had normal human insulin and Cpeptide secretion profiles during an iPGTT (Fig. S10F-S10H). In contrast, mice transplanted with hESC HNF1A KO $\beta$-cells did not increase human C-peptide secretion (Fig. 5G) after becoming severely diabetic (blood glucose $>500 \mathrm{mg} / \mathrm{dl}$ ) within one week following STZ injection (Fig. 5H). Consistent with in vitro studies, hESC HNF1A KO $\beta$-cells are unresponsive to blood glucose even at high levels, failing to maintain systemic glucose homeostasis in a mouse model of $\beta$-cell deficient diabetes.

HNF1A R200Q homozygous mutation is pathogenic and causes a developmental bias towards the $\alpha$-cell fate in vitro

These studies demonstrate the molecular, cellular and functional consequences of complete HNF1A deficiency, a situation that does not occur clinically, but was created experimentally to amplify the effects of HNFIA functional hypomorphism in order to enable mechanistic studies. 
MODY3 patients are heterozygous for the causal HNF1A alleles; to more directly characterize the consequences of heterozygous patient-specific mutations in $H N F 1 A$, we differentiated two heterozygous MODY3 iPSC lines (+/460_461insCGGCATCCAGCACCTGC and +/R200Q), isogenic mutation-corrected MODY3 iPSC lines (R200Q corrected: WT) and hESC HNF1A Het line into endocrine cells. Heterozygous mutations in $H N F 1 A$ did not affect the generation of definitive endoderm cells ( $\mathrm{SOX} 17^{+}$) at day 3 of differentiation or pancreatic progenitor cells $\left(\mathrm{PDX}^{+} / \mathrm{NKX} 6.1^{+}\right)$at day 11 of differentiation compared to WT lines as determined by immunohistochemistry (Fig. S11A). At the endocrine stage (day 27), cell clusters or organoids were morphologically indistinguishable and all heterozygous cell lines differentiated efficiently to all pancreatic endocrine cells ( $\operatorname{sc} \alpha-$, sc $\beta$ - and $\operatorname{sc} \delta$-like cells) and $\operatorname{sc} \beta$-like cells co-expressed PDX1/NKX6.1/HNF1A (Fig. S11B). HNF1A protein was detected in WT, MODY3 R200Q Het (+/R200Q) and MODY3 R200Q-corrected WT (+/+) iPSC-derived endocrine cells by western blot (Fig. S11C). In contrast to $H N F 1 A$ truncated lines, the heterozygous point mutation R200Q of MODY3 iPSC line did not affect the total amount of HNF1A protein, suggesting that the mutant R200Q protein was produced (Fig. S11C). No significant differences were found in endocrine cell types (sc $\alpha$-, sc $\beta$ - and $\operatorname{sc} \delta$-like cells) between $H N F 1 A$ WT and heterozygous cell lines at day 27 of differentiation by immunohistochemistry (Fig. S11D).

To determine the consequences of heterozygosity for HNF $1 A$ in our MODY3 patient lines for the bias towards $\alpha$-cell fate, we measured the percentage of glucagon cells co-expressing C-peptide in two MODY3 iPSC Het (+/460ins and +/R200Q) lines in vitro. We found no significant differences between MODY3 iPSC lines and control iPSC R200Q-corrected WT lines (Fig. S11E). To determine the pathogenicity of the $H N F 1 A$ R200Q mutation, we knocked out of the $H N F 1 A$ 
wildtype allele in a MODY3 iPSC Het line (R200Q/-), which resulted in a significant $\sim 70 \%$ increase in GCG and CPEP co-expressing cells compared to the R200Q-corrected WT control lines (Fig. S11E and S11F). We also detected a significant increase in the percentage of GCG and CPEP co-expressing cells in hESC HNF1A R200Q homozygous (R200Q/R200Q) and hESC HNF1A Het (+/-) lines compared to hESC WT (+/+) lines (Fig. S11G and S11H). Thus, while both heterozygous MODY3 patient cells (+/460ins and +/R200Q) show no developmental bias towards the $\alpha$-cell fate, complete loss of HNF1A WT allele in MODY3 iPSC R200Q (R200Q/-) and hESC HNF1A R200Q homozygous (R200Q/R200Q) result in a stronger developmental bias towards $\alpha$-cell fate, indicating the pathogenicity of R200Q mutation. Interestingly, the hESC heterozygous line harboring a frameshift mutation in the DNA-binding domain and causing a premature stop codon presented a stronger bias towards $\alpha$-cell fate. Previous studies in MODY3 patients have shown that frameshift mutations in the DNA-binding domain cause dominantnegative effects affecting the DNA binding capacity of the normal HNF1A (43).

\section{HNF1A haploinsufficiency gradually impairs sc $\beta$-cell functional capacity in the context of}

\section{metabolic stress}

In order to assess the developmental potential of MODY3 patient-specific mutations in vivo, we transplanted pancreatic islet-like clusters derived from iPSCs using identical methods as previously described. While $87.9 \%$ (51/58) of mice transplanted with isogenic MODY3 iPSC Het lines (+/R200Q) and R200Q-corrected WT $(+/+)$ were teratoma-free; the majority $(61.5 \%, 8 / 13)$ of the MODY3 iPSC Het (460ins) and control iPSC WT lines showed teratoma formation (Fig. S11I). Because of variable teratoma formation among different iPSC lines (20), only teratomafree mice were used for further analysis. Teratoma-free transplanted mice had $67.3 \%(35 / 52)$ 
engraftment efficiency. Thirty weeks post-transplantation, we isolated grafts from normoglycemic animals (Fig. S11J) and found no significant differences in glucagon-to-insulin content ratios or endocrine cell types between MODY3 iPSC Het (+/R200Q) and MODY3 iPSC R200Q-corrected WT $(+/+)$ control grafts (Fig. S11K and S11L). These results are consistent with our iPSC in vitro results; however, a recent study from cadaveric MODY3 human islets with a T260M heterozygous mutation in DNA-binding domain (29), showed a bias of endocrine cells toward the $\alpha$-cell fate (Fig. 2K). The same phenotype was observed in hESC HNF1A heterozygous lines with frameshift mutation in the DNA-binding domain (Fig. S11H). Mutations affecting the DNA-binding domain or truncating mutations are associated with earlier onset of diabetes in MODY3 patients (44). Thus, different mutations affect HNF1A function to different degrees, resulting in different phenotypes in patients as well as in cellular phenotypes in a stem cell system.

To understand the function of MODY3 iPSC Het $\beta$-cells in a physiological context, we monitored circulating human insulin and C-peptide in transplanted euglycemic mice over 30 weeks. Mice transplanted with iPSC-derived cells from heterozygous MODY3 patient (+/460_461insCGGCATCCAGCACCTGC) had low, but detectable circulating human C-peptide two, four, eight (Fig. S12A) and thirty weeks post transplantation (Fig. S12B) with undetectable human insulin (Fig. S12C). Since human insulin levels are under the minimal threshold limit for detection by ELISA, this deficiency can be attributable to the lower ability of these mutant iPSCs to generate functional insulin producing cells in vivo. In mice transplanted with iPSC-derived cells from MODY3 patient (+/R200Q) and R200Q-corrected WT (+/+) isogenic lines, circulating human C-peptide (Fig. S12B) and human insulin (Fig. S12C) concentrations reached similar levels 30 weeks post-transplantation. Transplantation of mice with hESC $H N F 1 A$ Het (+/-) and hESC 
HNF1A R200Q homozygous (R200Q/R200Q) line resulted in low circulating concentrations of human C-peptide over thirty weeks post transplantation (Fig. S12A and S12B) with low to undetectable human insulin in hESC HNF1A Het (+/-) and hESC HNF1A Hom (R200Q/R200Q) as compared to WT lines (Fig. S12C), thus affecting insulin:C-peptide secretion ratios. These results show that $H N F 1 A$ heterozygous frameshift mutations in the DNA-binding domain (hESC +/-) and HNF1A R200Q homozygosity (hESC R200Q/R200Q) are pathogenic and that in the heterozygous point mutations (iPSC $+/ \mathrm{R} 200 \mathrm{Q}$ ), it is less detrimental to $\beta$-cell function.

To further interrogate the consequence of the R200Q mutation in $\beta$-cells function and evaluate glucose-stimulated insulin secretion, we performed an intraperitoneal glucose tolerance test (iPGTT) in mice transplanted with the MODY3 isogenic lines (R200Q Het and R200Q-corrected WT), as these iPSC lines allowed the reliable generation of transplanted mice with high C-peptide and insulin levels over thirty weeks post transplantation (Fig. S12D and S12E). During an iPGTT, mice transplanted with both isogenic lines showed comparable changes in blood glucose (Fig. S12F) and plasma human C-peptide (Fig. 6A); plasma human insulin concentrations dropped below detection during fasting and increased upon glucose injection (Fig. 6B), demonstrating homeostatic glucose responsiveness. Glibenclamide injection increased C-peptide and insulin concentrations in both genotypes (Fig. S12G and S12H) at equivalent ratios (Fig. S12I). No significant differences in insulin secretion (Fig. S12J) or endocrine hormone content were found in vitro or in vivo between genotypes (Fig. S12K and S12L).

MODY3 patients generally display normal glucose tolerance during early childhood and exhibit symptomatic diabetes only in their late teens or early adulthood (9) depending on the type and 
position of the $H N F 1 A$ mutation (44), indicating that progression of the disease occurs over several years and potentially involves metabolic stressors. To determine whether there are functional differences between heterozygous MODY3 mutated (+/R200Q) and MODY3 R200Q-corrected WT (+/+) sc $\beta$-cells, we treated mice with STZ, thereby exposing transplanted $\operatorname{sc} \beta$-cells to higher insulin demand due to ablation of mouse endogenous $\beta$-cells. Two weeks post STZ, animals transplanted with MODY3 iPSC R200Q Het (+/R200Q) cells showed rapid progression to hyperglycemia (Fig. 6C). The increase in blood glucose from those mice $(n=9)$ was accompanied by a failure to increase circulating human C-peptide (Fig. 6D) and a significant reduction of human insulin levels (Fig. 6E) over 5-6 weeks after STZ treatment. In contrast, in mice (n=6) transplanted with isogenic MODY3 iPSC R200Q-corrected WT cells (+/+), concentrations of human plasma C-peptide (Fig. 6D) and insulin increased (Fig. 6E).

Five to six weeks post STZ treatment, we found that the ratio of circulating insulin to C-peptide fell by 55\% in mice transplanted with MODY3 iPSC R200Q Het (+/R200Q) cells. In contrast, insulin:C-peptide ratios remained constant in mice transplanted with MODY3 R200Q-corrected WT $(+/+)$ cells $($ Fig. 6F). The decrease in circulating insulin:C-peptide ratios was reciprocal to a significant increase by $74 \%$ in the insulin:C-peptide ratios of intracellular content from isolated HNF1A mutated grafts compared to isogenic controls (Fig. 6G), demonstrating complementary imbalance of insulin to C-peptide secreted. These results show that HNF1A R200Q haploinsufficiency gradually impairs the stoichiometry of circulating insulin:C-peptide, and that gene correction of the $\mathrm{R} 200 \mathrm{Q}$ mutation protects MODY3 iPSC $\beta$-cells from acquiring this imbalance. 
We then performed iPGTTs two, four, six and eight weeks post STZ administration. MODY3 iPSC R200Q Het (+/R200Q) sc $\beta$-cells showed progressive impairment of glucose-stimulated insulin secretion: mild at two weeks post STZ (Fig. S13A and S13B) and completely unresponsive insulin release to glucose challenges four weeks post STZ (Fig. S13C and S13D). Mice transplanted with MODY3 sc $\beta$-cells fail to clear glucose from the circulation during a glucose tolerance test at six to eight weeks post STZ treatment (Fig. 6H and S13E). HNF1A R200Q heterozygous (+/R200Q) sc $\beta$-cells had no glucose-stimulated insulin secretion, whereas MODY3 R200Q-corrected WT $(+/+) \operatorname{sc} \beta$-cells were sensitive to fasting, remained glucose responsive six to eight weeks post STZ treatment, and cleared glucose from circulation (Fig. 6H-6I and S13E-S13F). Mice transplanted with MODY3 iPSC R200Q Het (+/R200Q) sc $\beta$-cells became diabetic as shown by elevated blood glucose (Fig. S13G) and HbA1c levels (Fig. 6J) compared to control mice. These results show that MODY3 iPSC $\beta$-cells (+/R200Q) fail to compensate for higher insulin demands, and that the hyperglycemia is due to gradual development of insulin secretory defects, characterized by a disruption of the stoichiometry of insulin and constitutive C-peptide release. Correction of R200Q mutations protects MODY3 iPSC $\beta$-cells from acquiring these abnormal insulin secretion profiles.

\section{Discussion}

Here we report the use of stem cell-derived islets containing $H N F 1 A$ mutations to elucidate the molecular basis for apparent $\beta$-cell dysregulation of insulin secretion in HNF1A hESC null and MODY3 hypomorphic patient lines. Based on in vitro and in vivo developmental, morphological and functional assessments of stem cell-derived islets, we conclude that $H N F 1 A$ mutations compromise calcium-mediated exocytosis of insulin-containing granules. In $H N F 1 A$ knockout stem cell-derived $\beta$-cells, glucose-stimulated insulin secretion is compromised in association with 
a reduction in CACNA1A and intracellular calcium levels, and down-regulation of SYT13, a gene involved in calcium-regulated granule exocytosis. The resulting retention and accumulation of insulin granules can be overcome through application of sulfonylureas, by increasing the low calcium levels found in HNF1A mutant cells. Thereby we demonstrate the molecular basis for the effective treatment of MODY3 with sulfonylureas. However, HNF1A deficiency has pleiotropic effects, which will need to be considered for effective treatment. The triggering of insulin release through sulfonylureas often becomes insufficient at later states of the disease.

In particular, HNF1A also has developmental functions; hypomorphic mutations in $H N F 1 A$ perturb expression of genes important in $\beta$-cell identity such as $P A X 4$, leading to increases in glucagon gene expression, $\alpha$-cell number and in the $\alpha: \beta$-cell ratio in mature islets. This phenotype was observed in homozygous hESC HNF 1A knockouts, but was not seen in MODY3 patient iPSCderived heterozygous cells (+/460ins and +/R200Q). This increase was, however, reproduced in MODY3 patient iPSC- and hESC-derived cells rendered homozygous for R200Q (iPSC R200Q/and in hESC R200Q/R200Q) indicating the pathogenicity of the R200Q allele. A recent case study of cadaveric human islets from a 33-year old MODY3 patient (heterozygous +/T260M mutation in the DNA-binding domain) with a 17-years history of diabetes also showed an increase in the ratio of $\alpha$-cell to $\beta$-cells (29).

The accumulation of abnormal insulin secretory granules in $H N F 1 A$ knockout $\beta$-cells was associated with a deviation of the 1:1 stoichiometric release of C-peptide and insulin from $\beta$-cells, characterized by constitutive release of C-peptide, and intracellular retention of insulin. Their equimolar secretion was restored by exposure of the cells to depolarization by sulfonylurea. 
Previous studies have identified a "constitutive-like" secretory pathway that, in immature $\beta$-cells, is characterized by accumulation of immature secretory granules that secrete newly synthesized C-peptide in molar excess of insulin $(45,46)$. The accumulation of abnormal insulin granules in $H N F 1 A$ deficient $\beta$-cells is apparently associated with an increase in insulin granule membrane remodeling, as a result of which C-peptide is diverted most likely to the constitutive-like secretion pathway, accounting for reduced insulin to C-peptide ratios detected in mice transplanted with HNF1A hypomorphic islets. Our data support this model, but more studies are necessary to validate this pathway with a detailed study of insulin secretory dynamics in MODY3 patients to fully elucidate the contribution of secretory defects described here. The observation in our study that Cpeptide present in the serum does not always involve equimolar secretion of insulin is clinically relevant when evaluating islet function in patients with MODY3. The ratio of insulin to C-peptide could be useful as a biomarker for progression of MODY3 and possibly other types of diabetes, pending more studies in patients.

The insulin secretory phenotype of heterozygous MODY3 iPSC-derived $\beta$-cells was intermediate to that of cells with complete HNF1A deficiency. MODY3 iPSC-derived heterozygous $\beta$-cells $(+/$ 200Q) initially functioned normally in transplanted mice, consistent with normal glucose tolerance of heterozygous carriers during early childhood, followed by symptomatic diabetes within the first three decades of life (Table S6 for age of onset) (9). Upon increasing the requirement for human insulin following ablation of the mouse's intrinsic $\beta$-cells, iPSC-derived $\beta$-cells from MODY3 patient (+/R200Q) were unable to fully compensate for the increased insulin requirement, and gradually developed phenotypes resembling those of $H N F 1 A$ knockout $\beta$-cells, including a failure to secrete insulin in response to glucose, and reduced insulin to C-peptide 
secretion ratios. Correction of the R200Q mutation in MODY3 iPSC-derived $\beta$-cells protected cells from acquiring abnormal insulin secretion profiles. This study provides direct evidence that the HNF1A R200Q mutation is functionally consequential and pathogenic, leading to insulin secretion defects.

Our studies demonstrate the utility of stem cell-based models in defining the molecular physiology of $\beta$-cell failure in gene-specific diabetes in humans. Specifically, we show pleiotropic effects of HNF1A deficiency at several levels of $\beta$-cell biology and function, consistent with the diversity of its transcriptional targets. Additional studies on this and other target genes(47) may provide novel targets for pharmacological interventions in MODY3. Among the clinical implications of this study are that stem cell derived human islet cell clusters are capable of supporting glucose homeostasis in mice. In MODY patients, $\beta$-cell autoimmunity is not mechanistically involved, so that islets created from somatic cells of such patients should be immunologically tolerated by the patient. As shown here, the mutant alleles of MODY3 (or other) patients could be corrected to produce autologous stem-cell derived islets with a normal $H N F 1 A$ allele. These cells could be used to restore glucose homeostasis in such subjects. 


\section{Material and methods}

Human subject and cell lines. Nine MODY3 subjects (Pt1 to Pt9), three MODY2 subjects (1068, 1133 and 1144) and four control subjects (1023, 1098, 1136 and 1015) were recruited at the Naomi Berrie Diabetes Center and monitored over 2-5 years. Samples were coded to protect subjects' identity (Fig. S2J). Biopsies from MODY3 subjects (Pt1, Pt2 and Pt3) and one control subject (1023) were cut into small pieces (approximately $5 \times 5 \mathrm{~mm}$ in size). 2-3 pieces of minced skin are placed next to a droplet of silicon in a well of six-well dish. A glass cover slip (22x22 mm) was placed over the biopsy pieces and silicon droplet. $5 \mathrm{ml}$ of biopsy plating media was added and incubated for 5 days at $37^{\circ} \mathrm{C}$. Biopsy pieces are then grown in culture medium for 3-4 weeks. Biopsy plating medium is composed of DMEM (Gibco, 10569), 10\% FBS (GE Healthcare, SH30088.03HI), 1\% GlutaMAX (Gibco, 35050061), 1\% Anti-Anti (Gibco, 15240062), NEAA (Gibco, 11140-050), 0.1\% 2-Mercaptoethanol (Gibco, 21985023) and nucleosides (Millipore ES008-D). Culture medium contains DMEM (Gibco, 10569), 10\% FBS (GE Healthcare, SH30088.03HI), 1\% GlutaMAX (Gibco, 35050061) and 1\% Pen-Strep (Gibco, 15070063). The hESCs (Mel1)(21) used in this manuscript is an NIH approved line. All research involving human subjects was approved by the Institutional Review Board of Columbia University Medical Center, and all participants provided written informed consent.

Generation of iPSCs and cytogenic analysis of stem cells. Primary fibroblasts were reprogrammed into pluripotent stem cells using CytoTune ${ }^{\text {TM}}$-iPS Sendai Reprogramming Kit (Invitrogen). 50,000 fibroblast cells (between passage 2-5) were seeded in a well of a six-well dish and allowed to recover overnight. The next day, cells were infected by Sendai virus expressing human transcription factors Oct4, Sox2, Klf4 and C-Myc mixed in fibroblast medium according 
to the manufacturer's instructions. Two days later, the medium was exchanged for human ES medium supplemented with the ALK5 inhibitor SB431542 (2 $\mu \mathrm{M}$; Stemgent), the MEK inhibitor PD0325901 (0.5 $\mu \mathrm{M}$; Stemgent), and thiazovivin (0.5 $\mu \mathrm{M}$; Stemgent). Human ES medium contained KO-DMEM (Gibco 10829), 15\% KnockOut Serum Replacement (Gibco, 10828), 1\% GlutMAX, 0.1\% 2-Mercaptoethanol, 1\% NEAA, 1\% PenStrep and $0.1 \mathrm{ug} / \mathrm{mL}$ bFGF (all from Gibco). On day 7-10 post infection, cells are detached using TrypLE ${ }^{\mathrm{TM}}$ Express (Gibco, 12605036) and passaged onto mouse embryonic fibroblast feeder cells (GlobalStem CF-1 MEF IRR). Individual colonies of induced pluripotent stem cells were manually picked between day 21-28 post infection and each iPSC line was expanded from a single colony. All stem cells lines are cultured on human ES medium. Cytogenic analysis performed on 20 G-banded metaphase cells from each line by Cell Line Genetics Inc (Fig. S2M).

Stem cell culture. hESC and iPSCs are grown on plates coated with primary mouse embryonic fibroblasts or MEFs (GlobalStem, CF-1 MEF IRR) and dissociated every 4-5 days using TrypLE ${ }^{\text {TM }}$ Express (Life Technology, 12605036) for passaging. After dissociation, cells are suspended in human ES medium containing $10 \mu \mathrm{M}$ ROCK inhibitor Y27632 (Selleckchem, S1049).

In silico gRNA design. sgRNAs (Table S1) were designed using an online CRISPR design tool (crispr.mit.edu) and cloned into a gRNA cloning vector (Addgene, 41824) following option B from the gRNA Synthesis Protocol(48). The resulting vector is transformed into competent bacteria using Gibson Assembly® chemical transformation protocol (E5510). Single clones are picked and grown on $3 \mathrm{ml}$ of LB broth for $16-18 \mathrm{~h}$ at $37^{\circ} \mathrm{C}$ in a shaker $(250 \mathrm{rpm})$; DNA is extracted and Sanger sequenced. 
CRISPR/Cas9 nucleofection and mutagenesis. Stem cells are cultured with human ES media containing ROCK inhibitor Y27632 (Selleckchem, S1049) 3h prior nucleofection, dissociated and filtered through a $70 \mu \mathrm{m}$ cell strainer (Thermo Fisher Scientific, 8-771-2). Approximately $2 \times 10^{6}$ cells are nucleofected (Lonza nucleofector, program A23) with $5 \mu \mathrm{g}$ of Cas9-GFP plasmid (Addgene, 44719), $5 \mu \mathrm{g}$ of sgRNA and $5 \mu \mathrm{g}$ of ssDNA donor template (Table S1) using Human Stem Cell Nucleofector ${ }^{\mathrm{TM}}$ kit 1 (Lonza, VVPH-5012) according to the manufactures protocol and cells replated. Cells are dissociated 48h later for GFP sorting using BD FACS Aria II cell sorter. As a quality control step, some unsorted cells were used to test the sgRNAs mutation efficiency using the Transgenomic SURVERYOR ${ }^{\circledR}$ mutation detection kit according to the manufactures protocol. Sorted cell are plated in a $10 \mathrm{~cm}$ dish and single clones are picked 7-10 days post sorting, clones are further expanded and DNA extracted for PCR using HNF1A primers (Table S1). Amplicons are sent for Sanger sequencing to GENEWIZ and clones with indels are further validated by TOPO ${ }^{\circledR}$ TA cloning (Thermo Scientific, 450641) (at least 6 clones are picked) followed by Sanger sequencing. For hESC WT line, five different clonal lines were used for analysis throughout the study.

Differentiation into pancreatic endocrine cells. Cells are grown to $80-90 \%$ confluency, dissociated and suspended in mTeSR TM medium (STEMCELL Technology, 05850) with $10 \mu \mathrm{M}$ ROCK inhibitor Y27632 (Selleckchem, S1049) and plated in a 1:1 ratio into Matrigel-coated (Fisher Scientific, 354277) wells for differentiation. Differentiation was performed using a published protocol (20). The initial stages of differentiation were conducted in planar culture (d0d11). For definitive endoderm stage (d1-d3) cells were cultured using STEMdiffTM Definitive 
Endoderm Differentiation Kit (Stemcell Technologies, 05110). For primitive gut stage (d4-d6), cells were cultured in RPMI containing GlutaMAX (Life Technology, 61870-127), 1\% (v/v) Penicillin-Streptomycin (PS) (Thermo Fisher Scientific, 15070-063), 1\% (v/v) B27 Serum-Free Supplement (50x) (Life Technology, 17504044) and 50 ng/ml FGF7 (R\&D System, 251-KG). For posterior foregut stage (d7-d8), cells were cultured in DMEM containing GlutaMax, $1 \%(\mathrm{v} / \mathrm{v})$ PS, 1\% (v/v) B27, $0.25 \mu \mathrm{M}$ KAAD-Cyclopamine (Stemgent, 04-0028), $2 \mu \mathrm{M}$ Retinoic acid (Stemgent, 04-0021) and 0.25 $\mu \mathrm{M}$ LDN193189 (Stemgent, 04-0074). For pancreatic progenitor stage (d9d11), cells were cultured in DMEM containing GlutaMax, 1\% (v/v) PS, 1\% (v/v) B27 and 50 ng/ml EGF (R\&D System, 236-EG). Cells were then dissociated using TrypLETM Express (Life Technology, 12605036) and seeded into low-attachment 96 well-plates (Corning, 7007) (1 well of 6 well-plate to 60 wells of 96-well-plate) for clustering step to form aggregates or clusters of endocrine cells in DMEM containing GlutaMax, 1\%(v/v) PS, 1\% (v/v) B27, $0.25 \mu \mathrm{M}$ Cyclopamine, $1 \mu \mathrm{M}$ thyroid hormone (T3) (Sigma, T6397), $10 \mu \mathrm{M}$ Alk5i, $10 \mu \mathrm{M}$ Zinc sulfate (Sigma-Aldrich, Z4750) and $10 \mu \mathrm{g} / \mathrm{ml}$ Heparin (Sigma-Aldrich, H3149) for 2 days (d12-d13). For pancreatic endocrine stage (d14-20) cells were cultured using DMEM containing GlutaMax, 1\% (v/v) PS, 1\% (v/v) B27, $100 \mathrm{nM}$ LDN, $1 \mu \mathrm{M} \mathrm{T3}, 10 \mu \mathrm{M}$ Alk5i, $10 \mu \mathrm{M}$ Zinc sulfate, $10 \mu \mathrm{g} / \mathrm{ml}$ Heparin and $100 \mathrm{nM}$ gamma-secretase inhibitor (DBZ) (EMD Millipore, 565789). For mature pancreatic endocrine stage (d21-d27) cells were cultured using DMEM containing GlutaMax, $1 \%$ (v/v) PS, 1\% (v/v) B27, $1 \mu \mathrm{M}$ T3, $10 \mu \mathrm{M}$ Alk5i, $10 \mu \mathrm{M}$ Zinc sulfate, $10 \mu \mathrm{g} / \mathrm{ml}$ Heparin, $1 \mathrm{mM} \mathrm{N}$ acetyl cysteine (N-Cys) (Sigma-Aldrich, A9165-5G), $10 \mu \mathrm{M}$ Trolox (EMD Millipore, 648471$500 \mathrm{MG}$ ) and $2 \mu \mathrm{M}$ R428 (Tyrosine kinase receptor AXL inhibitor) (ApexBio, A8329). From d1 to $\mathrm{d} 11$ media was changed every day and from $\mathrm{d} 12$ to $\mathrm{d} 27$ media was changed every other day. All differentiations were done for 27 to 30 days. 
In vitro insulin secretion and content. Static insulin secretion assay was performed at day 27-30 of differentiation; 10 islet-like clusters of cells were used per experiment. Islet-like clusters were pre-incubated for 1 hour in Kreb's Ringer Buffer (128mM NACL, 5mM KCL, 2.7mM CaCl2, 1.2mM MgSO4, 1mM NaHPO4, 1.2mM KH2PO4, 5mM NaHCO3, 10mM HEPES, 0.1\% Bovine Serum Albumin, $\mathrm{pH}=7.4$ ) containing $3.3 \mathrm{mM}$ glucose, washed and incubated for another hour in $3.3 \mathrm{mM}$ glucose and the medium was collected. Subsequently, $200 \mu 1$ of buffer containing 16.7 $\mathrm{mM}$ glucose or $400 \mu \mathrm{M}$ tolbutamide (abcam, ab120278) or $30.5 \mathrm{mM} \mathrm{KCl}$ was used to treat cells for 1 hour, after which the medium was collected. Insulin content was measured by acid ethanol extraction; cells are resuspended in $50 \mu 1$ of water and sonicated for 15 seconds. The sonicate is mixed with acid ethanol $(0.18 \mathrm{M} \mathrm{HCl}$ in $96 \%$ ethanol (vol/vol) $)$, in a 1:3 ratio of sonicate to acid ethanol. The mixed solution is incubated at $4^{\circ} \mathrm{C}$ for 12 hours. Human C-peptide, human insulin and human proinsulin secretion and content was measured using Ultrasensitive C-peptide ELISA (Mercodia, 10-1141-01), Insulin ELISA (Mercodia, 10-1113-01) and Proinsulin ELISA (Mercodia, 10-1118-01) kit according to the manufacturer's protocol. Mouse insulin was measured using Ultrasensitive Insulin ELISA (Mercodia, 10-1249-01) kit according to the manufacturer protocol. All samples were handled the same way.

Dynamic insulin secretion. Perifusion was performed as described previously(49), 20 randomly chosen cluster of cells were examined using a Biorep Technologies (Miami, FL) perifusion system. Clusters were perifused with Kreb's buffer $[115 \mathrm{mM} \mathrm{NaCl}, 5 \mathrm{mM} \mathrm{KCl}, 24 \mathrm{mM} \mathrm{NaHCO}$, $1 \mathrm{mM} \mathrm{MgCl} 2,2.2 \mathrm{mM} \mathrm{CaCl}_{2}$ at $\mathrm{pH}$ 7.4] supplemented with $0.17 \%$ bovine serum albumin and 3.3 $\mathrm{mM}$ glucose (26 min), followed by $16.7 \mathrm{mM}$ glucose (35 min), $3.3 \mathrm{mM}$ glucose (15 min), $400 \mu \mathrm{M}$ 
tolbutamide in $3.3 \mathrm{mM}$ glucose ( $35 \mathrm{~min}), 20 \mathrm{mM} \mathrm{KCl}$ plus $3.3 \mathrm{mM}$ glucose (10 min) and finally $3.3 \mathrm{mM}$ glucose $(15 \mathrm{~min})$. Medium was collected at a flow rate $100 \mu 1 / \mathrm{min}$ to assess insulin secretion. Insulin concentration was measured using an Insulin ELISA kit (Alpco, 80-INSHUE01.1). Cluster of cells were collected at the end of the study and placed in acidified ethanol overnight to determine total insulin levels. All samples were handled the same way.

Calcium Imaging. Stem cell-derived $\beta$-cells were dissociated from clusters and plated onto 35 mm glass-bottom dishes coated with 5\% Matrigel (Fisher Scientific, 354277). Cells were washed twice with basal KRBH solution composed of (mM): $128 \mathrm{NACl}, 5 \mathrm{KCl}, 2.7 \mathrm{CaCl}_{2}, 1.2 \mathrm{MgSO} 4,1$ $\mathrm{NaHPO}_{4}, 1.2 \mathrm{KH}_{2} \mathrm{PO}_{4}, 5 \mathrm{NaHCO}_{3}, 10$ HEPES, 0.1\% Bovine Serum Albumin, 3.3 glucose, $\mathrm{pH}$ to 7.4. Cells were then incubated in the same solution containing $1 \mathrm{uM}$ fura-2, AM (ThermoFischer, F1221) with $0.05 \%$ pluronic F-127 in DMSO (ThermoFischer, P3000MP) for $15 \mathrm{~min}$. at $37^{\circ} \mathrm{C}, 5 \%$ $\mathrm{CO}_{2}$. Cells were washed twice with basal $\mathrm{KRBH}$ solution and then imaged on an inverted Nikon Ti-eclipse microscope with a Nikon Plan fluor 20x objective (0.45 N.A.). Fura-2 measurements were collected at excitation wavelengths of 340 and $380 \mathrm{~nm}$ using EasyRatioPro (HORIBA Scientific). Stimulation solutions included either $16.7 \mathrm{mM}$ glucose, $600 \mu \mathrm{M}$ tolbutamide, or 30.5 $\mathrm{mM} \mathrm{KCl}$, with $\mathrm{NaCl}$ concentrations adjusted accordingly to balance osmolarity with $\mathrm{KRBH}$ solution.

Calcium concentrations were calculated as follows:

$$
\left[\mathrm{Ca}^{2+}\right]=K_{d} \cdot \frac{R-R_{\min }}{R_{\max }-R} \cdot \frac{S_{f 2}}{S_{b 2}}
$$


where $\mathrm{K}_{\mathrm{d}}$, the apparent $\mathrm{Ca}^{2+}$ binding affinity to Fura-2, was assumed to be $225 \mathrm{nM}$ and $R_{\max }, R_{\min }$, $S_{\mathrm{f} 2}$, and $S_{\mathrm{b} 2}$ values were obtained using $10 \mu \mathrm{m}$ ionomycin with either no $\mathrm{Ca}^{2+}$ or $2 \mathrm{mM} \mathrm{Ca}^{2+}$, values $(50,51)$

Immunohistochemistry. Clusters of cells and tissue (graft or pancreas) were fixed with $4 \%$ paraformaldehyde for 20 minutes at room temperature or $24 \mathrm{~h}$ at $4^{\circ} \mathrm{C}$ respectively, washed in $\mathrm{PBS}$, then dehydrated with $30 \%$ sucrose for 24 hours. Cells and tissues were washed in PBS, then cryopreserved in frozen OCT and sectioned at $7 \mu \mathrm{m}$ on microscope slides. Slides are then incubated with blocking solution containing 3\% donkey serum with $0.1 \%$ triton X-100 diluted in PBS for $1 \mathrm{~h}$ at room temperature. Primary antibodies (Table S7) are added in block solution overnight at $4^{\circ} \mathrm{C}$, washed three times for 5 minutes in PBST (PBS with $0.1 \%$ Tween-20) and incubated with secondary antibodies (Table S7) diluted in block solution for $1 \mathrm{~h}$ at room temperature, washed three times in PBST containing $10 \mu \mathrm{g} / \mathrm{ml}$ DAPI for 5 minutes and mounted with a coverslip in fluorescent mounting media (Dako, S3023). All images were taken using a Zeiss LSM 710 confocal microscope and quantified manually using ImageJ software. Quantification of cell type at the definitive and pancreatic progenitor stage (planar culture) were done by randomly choosing 4 sections in the well and averaging the percentage of cells from all sections. For cluster of cells (3D culture) and tissue (graft or pancreas), they were cut in 3-6 sections spaced $20 \mu \mathrm{m}$ and $100 \mu \mathrm{m}$ respectively and cell type was quantified in each section as a percentage. Final quantification result is the average of all sections. The ratio of hormone-positive cells for each insulin and glucagon over the total number of cells identified by DAPI nuclear staining was quantified using imageJ and reported as the fraction of total pancreatic parenchyma for each hormone (i.e. hormone-positive area). Each cluster of cell is comprised of $\sim 10^{\prime} 000$ cells. 
Flow Cytometry. Clusters of endocrine cells are dissociated into single cells using TrypLE ${ }^{\mathrm{TM}}$ Express (Life Technology, 12605036). Cells are then fixed with 4\% paraformaldehyde for 20 minutes at room temperature followed by 10 minutes permeabilization with cold methanol at $20^{\circ} \mathrm{C}$. Cells are washed with $3 \%$ donkey serum diluted in PBS and primary antibodies diluted (Table S7) in blocking solution containing 3\% donkey serum with $0.1 \%$ triton $\mathrm{X}-100$ diluted in PBS overnight at $4^{\circ} \mathrm{C}$. For tunel assay (Biotium, 30074) cells are incubated with fluorescent biotinylated nucleotide conjugate according to the manufacturer's instructions. Cells are washed in PBST (PBS with 0.1\% Tween-20) and incubated with secondary antibodies diluted (Table S7) in block solution for $1 \mathrm{~h}$ at room temperature. Cells are washed and filtered with a BD Falcon $12 \times 75-\mathrm{mm}$ tube with a cell strainer cap (BD Biosciences, 352235) and analyzed by flow cytometer. Data were analyzed using FlowJo software. Gating for flow cytometry was determined by incubating the cells only with secondary antibodies (negative control) and was consistent across experiments (shown in magenta in figures).

Western Blot. Cluster of endocrine cells are dissociated into single cells using TrypLE ${ }^{\text {TM }}$ Express (Life Technology, 12605036). Cells are lysed using RIPA buffer (NP40 1\%, NaCl 150mM, EDTA 1mM, Tris-HCl pH 7.5 50nM, SDS 0.1\%, Sodium Deoxychol 0.5\%, NaF 10mM, protease inhibitor tablet) and whole-cell lysates obtained by subsequent centrifugation. Immunoblots were incubated with primary antibodies against HNF1A (abcam, ab96777, 1:500) and $\beta$-Tubulin-III (Sigma, T2200, 1:500) (Table S7). 
Bulk RNA sequencing. Around 200 clusters of cells are dissociated and sorted for GFP using BD FACS Aria II cell sorter. Sorted cells are pelleted by centrifugation and RNA extracted using total the RNA purification micro (Norgen Biotek, 35300) kit according to the manufactures protocol. RNA quality and concentration is determined by an Agilent bioanalyzer and RNAseq performed by the Columbia University Genome Center. A poly-A pull-down was used to enrich mRNAs from total RNA samples (200ng-1ug per sample, RIN $>8$ required) and proceed on library preparation by using Illumina TruSeq RNA prep kit. Libraries are then sequenced using an Illumina HiSeq2000. RTA (Illumina) was used for base calling and bcl2fastq (version 1.8.4) for converting BCL to fastq format, coupled with adaptor trimming. The reads were mapped to a reference genome (Human: NCBI/build37.2; Mouse: UCSC/mm9) using Tophat(52) (version 2.1.0) with 4 mismatches $(--$ read-mismatches $=4)$ and 10 maximum multiple hits $(--$ max-multihits $=10)$. To tackle the mapping issue of reads that are from exon-exon junctions, Tophat infers novel exonexon junctions ab initio, and combine them with junctions from known mRNA sequences (refgenes) as the reference annotation. The relative abundance (aka expression level) of genes and splice isoforms is estimated using cufflinks(53) (version 2.0.2) with default settings. Differentially expressed genes are tested under various conditions using DEseq(54). An R package based on a negative binomial distribution that models the number reads from RNA-seq experiments to test for differential expression.

Single-cell RNA sequencing. Around 100 clusters of cells per genotype at day 27 of differentiation are dissociated and sorted for GFP using BD FACS Aria II cell sorter. Single cell RNA-Seq data from total of 271 hESC-derived cells (113 for WT and 158 for KO) were obtained. Raw reads were aligned to the hg19 reference genome using STAR (2.5.2b) and gene 
quantification were performed using FeatureCounts (1.5.2). Quality control was conducted as follows: cells with low mapped reads $(<1,000,000)$, low exon count $(<100,000)$, low exon mapped rate $(<0.2)$, high intergenic mapped rate $(>0.3)$, low detected gene count $(<1,000)$ and with more than $10 \%$ Mitochondria genes' count were removed. In addition, genes with $<10$ non-zero counts across all the cells were eliminated. All the downstream analysis was done with in R-3.4.1. Filtered raw count data were normalized and scaled by Seurat package and only variable genes generated by Seurat were used to do PCA and t-SNE. $n=3$ for each genotype.

10X genomics single-cell RNA sequencing. Around 100 clusters of cells per genotype at day 27 of differentiation are dissociated. Single cells were suspended in PBS $+0.04 \%$ BSA. Cellular suspensions ( $\sim 6,000$ cells) were loaded on a Chromium Single Cell Instrument (10X Genomics) to generate single cell GEMs. Single-cell RNAseq libraries were prepared using Chromium Single cell $3 \not$ Library, Gel beads \& Mutiplex kit (10X Genomics). Sequencing was performed on Illumina NextSeq500 using the following read length: 59-bp Read1 for transcript read, 14-bp I7 Index for Cell Barcode read, 8-bp I5 Index for sample index read, and 10-bp Read2 for UMI read. The samples were sequenced in ten different libraries (batches). Among all these samples, Batch 980379 and Batch 993091 (2 libraries) are HNF1A mutants (R200Q/R200Q), Batch 980380 and Batch 993090 (2 libraries) are HNF1A knockouts (KO) while Batch 989516 and Batch 993089 (2 libraries) are HNF1A wildtype cells (WT). In addition, to increase the sequencing depth, each batch was sequenced from 7 flowcells. The raw fastq files was processed via 10X Cell Ranger pipeline (cellranger-2.1.1). To note that GRCh38 reference genome was applied for the whole analysis. "cellranger count" with "--expect-cells=3,000" was run for each library respectively and "cellranger aggr" with default parameters was run to aggregate results generated from multiple 
libraries. A normalized umi-count matrix for all the cells was then generated. As for the quality control, cell and gene filtering process, cells with umi-count $<5,000$ and umi-count $>40,000$ and cells with $<2,000$ detected genes were removed. Also, genes with $<\sim 1 \%$ (25) non-zero counts across all the cells were eliminated from the analysis. To get rid of the Mitochondria cells, cells with more than $10 \%$ Mitochondria genes' count were also removed. All the downstream analysis was done with Seurat package in R-3.4.1. Every batch is an independent differentiation (biological replicates). $\mathrm{n}=2$ for each genotype.

Accession numbers. Single cell RNA sequencing and bulk RNA sequencing data were deposited in NCBI's Gene Expression Omnibus (GEO) database and accession number is GSE128331 and GSE129653.

Cell transplantation and in vivo assays. After 27-30 days of differentiation, $50 \mu \mathrm{g}$ of stem cellderived pancreatic cell clusters $(\sim 180$ islet-like clusters of $300-350 \mu \mathrm{m}$ diameter; each cluster comprised $\sim 10,000$ cells in total) including all islet endocrine cell types were suspended in $30 \mu 1$ of ice-cold Matrigel (Fisher Scientific, 354277) and loaded into ice-cold 1ml syringe with a $21 \mathrm{G}$ needle for transplantation. 6-10 weeks old male immunodeficient NSG (NOD.Cg-Prkdcscid Il2rgtm1Wj1/SzJ, Stock No: 005557, The Jackson Laboratories) mice were transplanted in the ventral and medial muscles (medial thigh and posterior lower) of the left thigh. Every 2 weeks post-transplantation, human C-peptide and human insulin was determined in the plasma of recipient mice at fed state (morning) for 30 weeks. An intraperitoneal glucose tolerance test was performed 18-24 weeks (before STZ injection) and 28-30 weeks (after STZ injection) posttransplantation by injecting $2 \mathrm{~g} / \mathrm{kg}$ body weight of 20\% D-glucose (Sigma, G8270) in PBS after 
overnight fasting (16-18h). For glibenclamide (Sigma, G0639) administration, 1mg/kg body weight of the drug diluted in PBS was i.p injected 24-28 weeks (before STZ injection) posttransplantation, all mice were at fed state but food was retrieved during the experiment. Blood was collected by tail bleeding in heparinized Eppendorf tubes at time points; plasma was isolated by centrifugation for 15 minutes at $2000 \mathrm{~g}$ at $4^{\circ} \mathrm{C}$. Human C-peptide was measured using Mercodia Ultrasensitive C-peptide ELISA (10-1141-01) and human insulin using Mercodia Insulin ELISA (10-1113-01) kit according to the manufactures protocol. All samples were handled the same way. Insulin detection limit is $1 \mathrm{mU} / \mathrm{L}$ as determined by the methodology described in the kit. Blood glucose levels were measured using a glucometer (FreeStyle Lite) and HbA1C using Siemens DCA 2000 Vantage Reagent kit (Siemens, 5035C). To induce diabetes, mice were i.p injected for 5 consecutive days with $40 \mathrm{mg} / \mathrm{kg}$ body weight of Streptozotocin (STZ) (Sigma-Aldrich, S0130$1 G)$ in PBS 24-28 weeks post-transplantation. All experiment procedures were performed according to Columbia University approved IACUC protocols.

Bioluminescence and fluorescence imaging. NSG mice transplanted with $G A P D H^{L u c i f e r a s e / w t}$ and $I N S^{G F P / w t}$ double reporter hESCs lines were i.p injected with $150 \mathrm{mg} / \mathrm{kg}$ body weight of D-luciferin potassium salt (Gold Biotechnology, luck-2G) in PBS 15 minutes before imaging on a IVIS spectrum optical imaging system (PerkinElmer). Signals were acquired with 1-minute exposure and analyzed using the Living image analysis software (Xenogen Corp.). Circular regions of interest (ROI) of the same size for all experiments were drawn around the signal in the left thigh and photons emitted over the time of exposure within the ROI measured. Bioluminescence was measured every 2 weeks post-transplantation for 30 weeks and after isolation of the graft at that 
time point. Luminescence was measured as described for bioluminescence after isolation of the graft. Background signals were subtracted from a nearby region and were consistent over time.

Electron microscopy. Fixed cells/tissues were incubated with $2.5 \%$ glutaraldehyde and $2 \%$ paraformaldehyde in $100 \mathrm{mM}$ sodium cacodylate buffer (pH 7.4) overnight. Samples were then treated with $1 \%$ osmium tetroxide in $100 \mathrm{mM}$ sodium cacodylate buffer for $1 \mathrm{~h}$, washed in distilled water four times (10 $\mathrm{min} / \mathrm{wash})$, and then treated with $2 \%$ aqueous uranyl acetate overnight at $4{ }^{\circ} \mathrm{C}$ in the dark. Samples were washed and sequentially dehydrated with increasing concentrations of acetone $(20,30,50,70,90$, and $100 \%)$ for $30 \mathrm{~min}$ each, followed by three additional treatments with $100 \%$ acetone for 20 min each. Samples were then infiltrated with increasing concentrations of Spurr's resin $(25 \%$ for $1 \mathrm{~h}, 50 \%$ for $1 \mathrm{~h}, 75 \%$ for $1 \mathrm{~h}, 100 \%$ for $1 \mathrm{~h}, 100 \%$ overnight at room temperature), and then incubated overnight at $70^{\circ} \mathrm{C}$ in a resin mold. Sections of 50-90 nm were cut on a Leica ultramicrotome with a diamond knife. Imaging was performed on an FEI Talos L120C operating at $120 \mathrm{kV}$.

Immunogold electron microscopy. 70nm thick sections from the embedded samples were placed on to carbon formvar 75 mesh nickel grids and etched using 4\% sodium metaperidotate for 10 minutes before being washed twice in distilled water and then blocked for 1 hour. Grids were incubated with the primary C-peptide antibody (Table S7) (1 in 10 dilution) for 1 hour at 4C overnight. Next day grids underwent seven washes in 1xPBS and then incubated in anti-rat $6 \mathrm{~nm}$ gold secondary (1 in 50 dilution) for 1 hour (Table S7). After this the grid was washed seven times in 1xPBS and twice in distilled water. Samples were then imaged on a ThermoFisher Talos L120C operating at $120 \mathrm{kV}$. 
Statistical analysis. All statistics were performed using Prism GraphPad software (La Jolla, CA). Normality of data set distributions was assessed by Shapiro-Wilk test and D'Agostino \& Pearson omnibus tests. Normally distributed data were analyzed by unpaired two-tailed $t$-test. For data not normally distributed we used a two-tailed Mann-Whitney test. Data are expressed as mean \pm standard error of the mean (SEM). $P<0.05$ was considered statistically significant. ${ }^{*} \mathrm{p}<0.05$, ${ }^{* *} \mathrm{p}<0.01,{ }^{* * *} \mathrm{p}<0.001 . \mathrm{n} . \mathrm{s}$ indicates a non-significant difference. 


\section{References and Notes:}

1. C. Pihoker et al., Prevalence, characteristics and clinical diagnosis of maturity onset diabetes of the young due to mutations in HNF1A, HNF4A, and glucokinase: results from the SEARCH for Diabetes in Youth. J Clin Endocrinol Metab 98, 4055-4062 (2013).

2. R. Naylor, A. Knight Johnson, D. del Gaudio, in GeneReviews((R)), M. P. Adam et al., Eds. (Seattle (WA), 1993).

3. T. M. Frayling et al., Mutations in the hepatocyte nuclear factor-1alpha gene are a common cause of maturity-onset diabetes of the young in the U.K. Diabetes 46, 720-725 (1997).

4. P. J. Kaisaki et al., Mutations in the hepatocyte nuclear factor-1alpha gene in MODY and earlyonset NIDDM: evidence for a mutational hotspot in exon 4. Diabetes 46, 528-535 (1997).

5. S. Ellard, K. Colclough, Mutations in the genes encoding the transcription factors hepatocyte nuclear factor 1 alpha (HNF1A) and 4 alpha (HNF4A) in maturity-onset diabetes of the young. Hum Mutat 27, 854-869 (2006).

6. A. Stride et al., Beta-cell dysfunction, insulin sensitivity, and glycosuria precede diabetes in hepatocyte nuclear factor-1alpha mutation carriers. Diabetes Care 28, 1751-1756 (2005).

7. M. M. Byrne et al., Altered insulin secretory responses to glucose in diabetic and nondiabetic subjects with mutations in the diabetes susceptibility gene MODY3 on chromosome 12. Diabetes 45, 1503-1510 (1996).

8. A. Stride et al., The genetic abnormality in the beta cell determines the response to an oral glucose load. Diabetologia 45, 427-435 (2002).

9. A. T. Hattersley, Maturity-onset diabetes of the young: clinical heterogeneity explained by genetic heterogeneity. Diabet Med 15, 15-24 (1998).

10. S. S. Fajans, G. I. Bell, K. S. Polonsky, Molecular mechanisms and clinical pathophysiology of maturity-onset diabetes of the young. N Engl J Med 345, 971-980 (2001).

11. M. Pontoglio et al., Defective insulin secretion in hepatocyte nuclear factor 1alpha-deficient mice. J Clin Invest 101, 2215-2222 (1998).

12. Z. Zhu et al., Genome Editing of Lineage Determinants in Human Pluripotent Stem Cells Reveals Mechanisms of Pancreatic Development and Diabetes. Cell Stem Cell 18, 755-768 (2016).

13. P. Wang et al., Combined Inhibition of DYRK1A, SMAD, and Trithorax Pathways Synergizes to Induce Robust Replication in Adult Human Beta Cells. Cell Metab 29, 638-652 e635 (2019).

14. L. Shang et al., beta-cell dysfunction due to increased ER stress in a stem cell model of Wolfram syndrome. Diabetes 63, 923-933 (2014).

15. H. Zeng et al., An Isogenic Human ESC Platform for Functional Evaluation of Genome-wideAssociation-Study-Identified Diabetes Genes and Drug Discovery. Cell Stem Cell 19, 326-340 (2016).

16. F. W. Pagliuca et al., Generation of functional human pancreatic beta cells in vitro. Cell 159, 428439 (2014).

17. A. Rezania et al., Reversal of diabetes with insulin-producing cells derived in vitro from human pluripotent stem cells. Nature biotechnology 32, 1121-1133 (2014).

18. M. C. Nostro et al., Efficient generation of NKX6-1+ pancreatic progenitors from multiple human pluripotent stem cell lines. Stem Cell Reports 4, 591-604 (2015).

19. J. R. Millman et al., Generation of stem cell-derived beta-cells from patients with type 1 diabetes. Nat Commun 7, 11463 (2016).

20. L. Sui et al., beta-Cell Replacement in Mice Using Human Type 1 Diabetes Nuclear Transfer Embryonic Stem Cells. Diabetes 67, 26-35 (2018).

21. S. J. Micallef et al., INS(GFP/w) human embryonic stem cells facilitate isolation of in vitro derived insulin-producing cells. Diabetologia 55, 694-706 (2012). 
22. M. Hara et al., Transgenic mice with green fluorescent protein-labeled pancreatic beta -cells. Am J Physiol Endocrinol Metab 284, E177-183 (2003).

23. S. Ma et al., beta Cell Replacement after Gene Editing of a Neonatal Diabetes-Causing Mutation at the Insulin Locus. Stem Cell Reports 11, 1407-1415 (2018).

24. D. Toaima et al., Identification of novel GCK and HNF1A/TCF1 mutations and polymorphisms in German families with maturity-onset diabetes of the young (MODY). Hum Mutat 25, 503-504 (2005).

25. V. Chandra et al., RFX6 regulates insulin secretion by modulating Ca2+ homeostasis in human beta cells. Cell Rep 9, 2206-2218 (2014).

26. Y. Xu et al., Generation and Phenotype Identification of PAX4 Gene Knockout Rabbit by CRISPR/Cas9 System. G3 (Bethesda) 8, 2833-2840 (2018).

27. E. Ganic, J. K. Johansson, H. Bennet, M. Fex, I. Artner, Islet-specific monoamine oxidase A and B expression depends on MafA transcriptional activity and is compromised in type 2 diabetes. Biochem Biophys Res Commun 468, 629-635 (2015).

28. B. Wu et al., Synaptotagmin-7 phosphorylation mediates GLP-1-dependent potentiation of insulin secretion from beta-cells. Proc Natl Acad Sci U S A 112, 9996-10001 (2015).

29. R. Haliyur et al., Human islets expressing HNF1A variant have defective beta cell transcriptional regulatory networks. J Clin Invest 129, 246-251 (2019).

30. T. Kuo et al., Induction of alpha cell-restricted $\mathrm{Gc}$ in dedifferentiating beta cells contributes to stress-induced beta-cell dysfunction. JCI Insight 5, (2019).

31. F. L. Cardenas-Diaz et al., Modeling Monogenic Diabetes using Human ESCs Reveals Developmental and Metabolic Deficiencies Caused by Mutations in HNF1A. Cell Stem Cell 25, 273-289 e275 (2019).

32. H. V. Petersen et al., Pax4 represses pancreatic glucagon gene expression. Mol Cell Biol Res Commun 3, 249-254 (2000).

33. S. A. Wells, Jr., J. C. Gunnells, J. D. Shelburne, A. B. Schneider, L. M. Sherwood, Transplantation of the parathyroid glands in man: clinical indications and results. Surgery 78, 34-44 (1975).

34. B. Sosa-Pineda, K. Chowdhury, M. Torres, G. Oliver, P. Gruss, The Pax4 gene is essential for differentiation of insulin-producing beta cells in the mammalian pancreas. Nature 386, 399-402 (1997).

35. B. K. Gage, R. K. Baker, T. J. Kieffer, Overexpression of PAX4 reduces glucagon expression in differentiating hESCs. Islets 6, e29236 (2014).

36. G. Song, G. Pacini, B. Ahren, D. Z. D'Argenio, Glucagon increases insulin levels by stimulating insulin secretion without effect on insulin clearance in mice. Peptides 88, 74-79 (2017).

37. T. M. Reinbothe et al., The human L-type calcium channel Cav1.3 regulates insulin release and polymorphisms in CACNA1D associate with type 2 diabetes. Diabetologia 56, 340-349 (2013).

38. C. Huang et al., Synaptotagmin 4 Regulates Pancreatic beta Cell Maturation by Modulating the $\mathrm{Ca}(2+)$ Sensitivity of Insulin Secretion Vesicles. Dev Cell 45, 347-361 e345 (2018).

39. J. S. Stancill et al., Chronic beta-Cell Depolarization Impairs beta-Cell Identity by Disrupting a Network of $\mathrm{Ca}(2+)$-Regulated Genes. Diabetes 66, 2175-2187 (2017).

40. Z. Gao, J. Reavey-Cantwell, R. A. Young, P. Jegier, B. A. Wolf, Synaptotagmin III/VII isoforms mediate Ca2+-induced insulin secretion in pancreatic islet beta -cells. J Biol Chem 275, 3607936085 (2000).

41. S. A. Andersson et al., Reduced insulin secretion correlates with decreased expression of exocytotic genes in pancreatic islets from patients with type 2 diabetes. Mol Cell Endocrinol 364, 36-45 (2012).

42. H. Yang, J. R. Wright, Jr., Human beta cells are exceedingly resistant to streptozotocin in vivo. Endocrinology 143, 2491-2495 (2002). 
43. J. M. Magana-Cerino et al., Identification and functional analysis of c.422_423InsT, a novel mutation of the HNF1A gene in a patient with diabetes. Mol Genet Genomic Med 5, 50-65 (2017).

44. C. Bellanne-Chantelot et al., The type and the position of HNF1A mutation modulate age at diagnosis of diabetes in patients with maturity-onset diabetes of the young (MODY)-3. Diabetes 57, 503-508 (2008).

45. R. Kuliawat, P. Arvan, Protein targeting via the "constitutive-like" secretory pathway in isolated pancreatic islets: passive sorting in the immature granule compartment. J Cell Biol 118, 521-529 (1992).

46. P. Arvan et al., Protein discharge from immature secretory granules displays both regulated and constitutive characteristics. J Biol Chem 266, 14171-14174 (1991).

47. F. L. Cardenas-Diaz et al., Modeling Monogenic Diabetes using Human ESCs Reveals Developmental and Metabolic Deficiencies Caused by Mutations in HNF1A. Cell Stem Cell 25, 273-289.e275 (2019).

48. P. Mali et al., RNA-guided human genome engineering via Cas9. Science 339, 823-826 (2013).

49. A. Jurczyk et al., Beyond the brain: disrupted in schizophrenia 1 regulates pancreatic beta-cell function via glycogen synthase kinase-3beta. FASEB J 30, 983-993 (2016).

50. G. Grynkiewicz, M. Poenie, R. Y. Tsien, A new generation of Ca2+ indicators with greatly improved fluorescence properties. J Biol Chem 260, 3440-3450 (1985).

51. J. Tong, T. V. McCarthy, D. H. MacLennan, Measurement of resting cytosolic Ca2+ concentrations and Ca2+ store size in HEK-293 cells transfected with malignant hyperthermia or central core disease mutant Ca2+ release channels. J Biol Chem 274, 693-702 (1999).

52. C. Trapnell, L. Pachter, S. L. Salzberg, TopHat: discovering splice junctions with RNA-Seq. Bioinformatics 25, 1105-1111 (2009).

53. C. Trapnell et al., Transcript assembly and quantification by RNA-Seq reveals unannotated transcripts and isoform switching during cell differentiation. Nat Biotechnol 28, 511-515 (2010).

54. S. Anders, W. Huber, Differential expression analysis for sequence count data. Genome Biol 11, R106 (2010). 
Figures

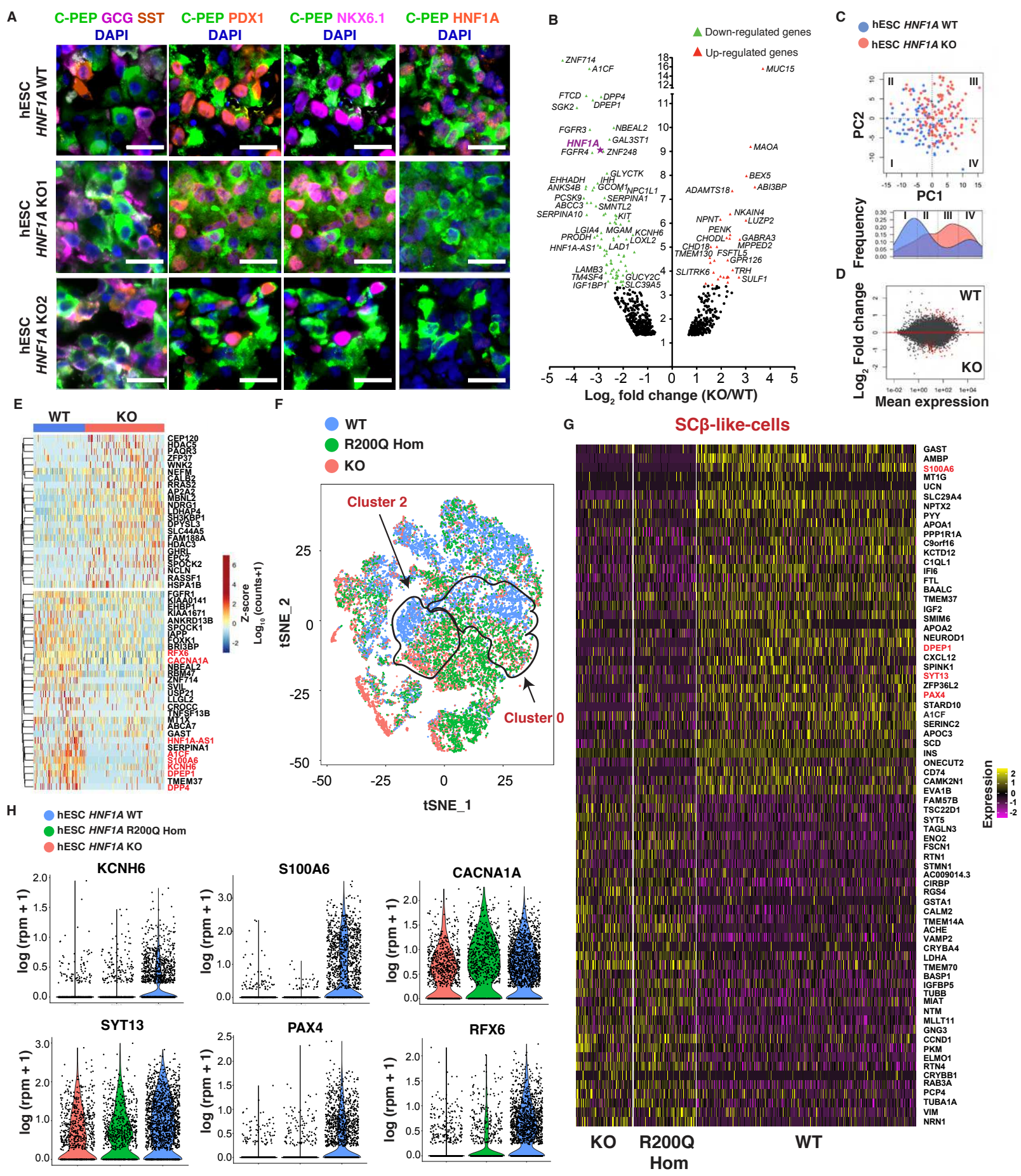

Fig. 1. HNF1A deficiency impairs a network of genes required for calcium signaling, glucose-

stimulated insulin exocytosis and $\beta$-cell fate in vitro. (A) Representative immunohistochemistry

(IHC) images of hESC-derived endocrine cell lines (HNF1A WT, KO1 and KO2) for indicated 
markers. White cells are GCG/CPEP double positive cells. Scale bars: $20 \mu \mathrm{m}$. (B-E) RNA sequencing of FACs sorted $I N S^{G F P / w t}$ positive cells between $H N F 1 A$ WT and KO genotypes (n=3 for both genotypes). (B) Volcano plot depicting fold change ( $\log _{2}$ fold change, $x$-axis) and statistical significance $\left(-\log _{10} \mathrm{p}\right.$-value, $\mathrm{y}$-axis) for differential gene expression (down-regulated in green; up-regulated in red) by bulk RNAseq (see also Table S3). (C) Cell clustering by principal component analysis (PCA). Lower panel depicts the radial distribution or frequency of individual cells from each PCA quadrant. (D) MA-plot depicting fold change ( $\log _{2}$ fold change, y-axis) and mean expression (counts, x-axis) of differentially-expressed genes. (E) Heatmap showing expression for each gene identified as z-score of expression from all sorted sc $\beta$-like cells. (F) Single cell RNA sequencing of 22164 (all genotypes combined) unsorted hESC-derived endocrine cells by HNF1A WT, R200Q homozygous and KO genotypes (n=3 for each genotype). Feature plot based on tSNE projection of cells where the colors denote different cell lines by $H N F 1 A$ genotype line via Louvain algorithm performed by Seurat. (G) Heatmap showing differentially expressed genes from sc $\beta$-like cells by $H N F 1 A$ WT, R200Q homozygous and KO genotypes. Total of $1846 \mathrm{sc} \beta$-like cells (all genotypes combined) were identified and displayed as $|\operatorname{logFC}|>0.35$ and adjusted $\mathrm{p}$-value $<1 \mathrm{e}^{-4}$. Genes are listed in decreasing order of $\log _{2}$ fold change between $H N F 1 A$ WT and HNF1A mutant genotypes. (H) Violin plot of cells based on KCNH6, S100A6, CACNA1A, SYT13, PAX4 and RFX6 gene expression $(\log 1(\mathrm{rpm}+1))$ from sc $\beta$-like cells. All stem cell differentiations were done for 27 days. (n) represents the number of biological replicates. See also Fig. S4 and S5. 


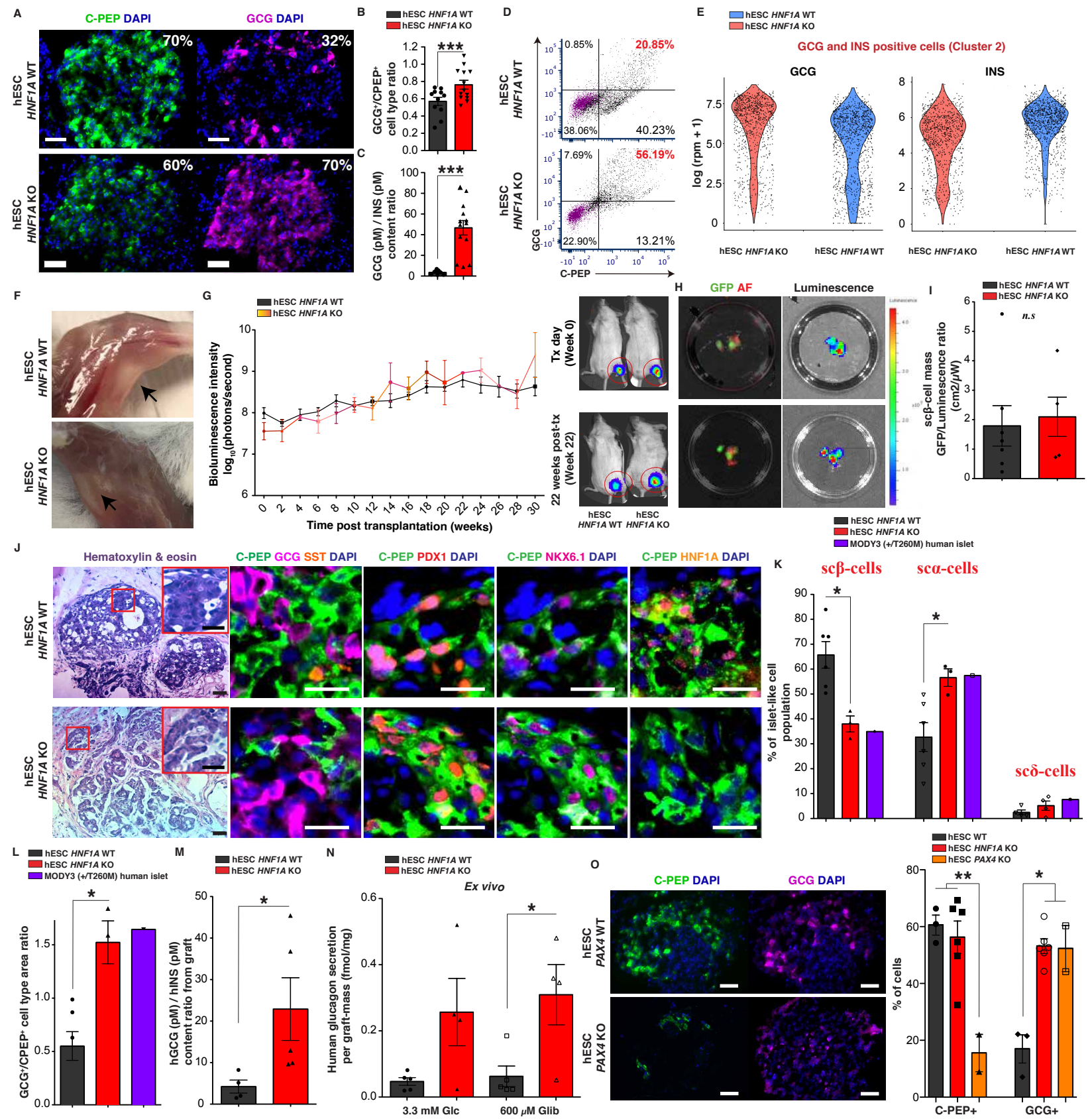

Fig. 2. HNF1A deficiency causes a developmental bias toward the $\alpha$-cell fate. (A)

Representative IHC images of hESC-derived endocrine cell lines (HNF1A WT and KO) for indicated markers. Scale bars: $50 \mu \mathrm{m}$. (B) $\mathrm{GCG}^{+} / \mathrm{CPEP}^{+}$cell type ratios by IHC and (C) GCG/INS protein content ratios measured by ELISA. p-values: ${ }^{* * *} \mathrm{p}<0.001$; two-tailed t-test. (D) $\mathrm{CPEP}^{+}$and $\mathrm{GCG}^{+}$populations by flow cytometry. Gating for GCG and CPEP negative cells (magenta) was determined by incubating cells without primary antibodies and with secondary antibodies. (E) 
Violin plot of total of 2670 (all genotypes) GCG and INS positive cells (cluster 2) displayed based on $G C G$ and $I N S$ expression $(\log (\mathrm{rpm}+1))(\mathrm{n}=3$ for each genotype). (F) Representative image of graft tissue (black arrows) 30 weeks post-transplantation with hESC-derived endocrine cells before explant from quadriceps muscle. (G) Bioluminescence intensity $\left(\log _{10}\right.$ photons/second) measured in mice transplanted with hESC-derived endocrine cells (WT n=11 and KO n=4) over time (weeks) with representative bioluminescence images. (H) Representative GFP fluorescence (sc $\beta$-cells) and bioluminescence images (total cells) (GFP in green; AF: tissue auto-fluorescence in red) from isolated grafts with (I) quantification of $\mathrm{sc} \beta$-cell mass GFP/luminescence ratio $\left(\mathrm{cm}^{2} / \mu \mathrm{W}\right)$. (J) Representative H\&E and IHC images showing hESC-derived endocrine cells for indicated markers. Scale bars: $20 \mu \mathrm{m}$. (K) Percentage of sc $\beta$-, sc $\alpha$ - and sc $\delta$-cells from the islet-like clusters and MODY3 islets $(H N F 1 A+/ \mathrm{T} 260 \mathrm{M})$ from Haliyur et al. (29). (L) $\mathrm{GCG}^{+} / \mathrm{CPEP}^{+}$cell type area ratios by $\mathrm{IHC}$ from isolated grafts and $\mathrm{GCG}^{+} / \mathrm{INS}^{+}$from MODY3 islets $(H N F 1 A+/ \mathrm{T} 260 \mathrm{M}$, Haliyur et al). Each point in plots is an islet-like cluster. Endocrine cell percentages were calculated as hormone-positive area from islet-like clusters across the entire graft (2 grafts for each genotype). (M) hGCG (pM)/hINS (pM) content ratio from isolated grafts. (N) Ex vivo human glucagon secretion normalized to graft mass (fmol/mg) in response to indicated secretagogues. All protein concentrations were measured by ELISA. (O) Representative IHC images of hESC-derived endocrine cell lines for indicated markers with quantification of CPEP+ and GCG+ cells (\%) by flow cytometry. All stem cell differentiations were done for $27-30$ days. 20 clusters $(\sim 10 \mathrm{k}$ cells per cluster) of endocrine cells were used flow cytometry. All mice were transplanted with clusters of hESC-derived endocrine cells (HNF1A WT and KO2) at day 27 of differentiation, and grafts were isolated 30 weeks post-transplantation for ex vivo analysis. (n) represents the number of biological replicates. For scatter plots, each point in plots represents an independent biological 
A STEM CELL MODEL OF HNF1A DEFICIENCY

experiment (n). Data are represented as mean \pm SEM. p-values: ${ }^{*} \mathrm{p}<0.05,{ }^{*} \mathrm{p}<0.01,{ }^{* * *} \mathrm{p}<0.001$; Mann-Whitney test. n.s: non-significant. See also Fig. S6 and S7. 

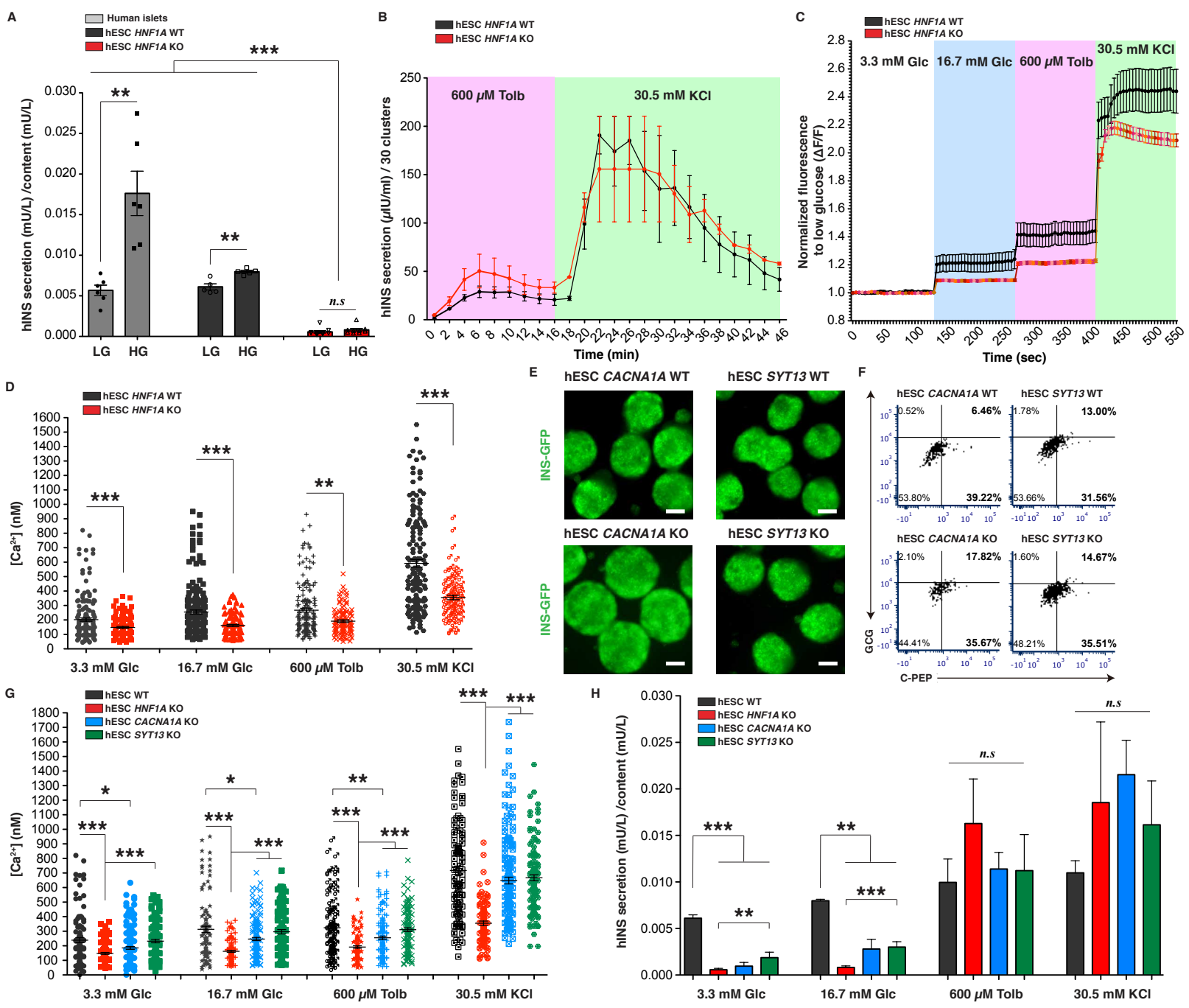

Fig. 3. HNF1A deficiency affects insulin secretion due to reduced intracellular calcium levels

in association with $C A C N A 1 A$ and SYT13 down-regulation in vitro. (A) Human insulin secretion $(\mathrm{mU} / \mathrm{L})$ in $1 \mathrm{~h}$ normalized to content $(\mathrm{mU} / \mathrm{L})$ in response to low glucose $(\mathrm{LG}, 3.3 \mathrm{mM})$ and high glucose (HG, $16.7 \mathrm{mM})$ stimulation in static assay of hESC-derived endocrine cell lines (HNF1A WT and KO) and pancreatic human islets. (B) Human insulin secretion $(\mu \mathrm{IU} / \mathrm{ml})$ normalized to 30 clusters in a perfusion assay (both $H N F 1 A$ WT and KO genotypes $\mathrm{n}=2$ ) in response to indicated secretagogues. (C) Intracellular calcium fluorescence normalized to low glucose (F340/380) from dispersed sc $\beta$-like cells with (D) absolute intracellular calcium concentration $(\mathrm{nM})$ quantification in response to indicated secretagogues (each point represents a 


\section{A STEM CELL MODEL OF HNF1A DEFICIENCY}

sc $\beta$-like-cell, $H N F 1 A$ WT $\mathrm{n}=170$ and $H N F 1 A$ KO n=110 cells). (E) Representative GFP-field images of endocrine organoids from indicated genotypes. Scale bars: $200 \mu \mathrm{m}$. (F) Representative $\mathrm{CPEP}^{+}$and $\mathrm{GCG}^{+}$populations quantification by flow cytometry. (G) Absolute intracellular calcium concentration $(\mathrm{nM})$ in response to indicated secretagogues (each point represents a sc $\beta$ like-cell from independent batches of differentiation, WT n=110, HNF1A KO n=110, CACNA1A KO n=175 and SYT13 KO n=120 cells). (H) Human insulin secretion (mU/L) in $1 \mathrm{~h}$ normalized to content $(\mathrm{mU} / \mathrm{L})$ in response to low $(3.3 \mathrm{mM})$ and high glucose $(16.7 \mathrm{mM})$ and indicated secretagogues in static assay (WT n=5, HNF1A KO n=9, CACNA1A KO n=6 and SYT13 KO n=5). All stem cell differentiations were done for 27 days. 20 clusters $(\sim 10 \mathrm{k}$ cells per cluster $)$ of endocrine cells were used flow cytometry. (n) represents the number of biological replicates. For scatter plots, each point in plots represents an independent biological experiment (n). Data are represented as mean \pm SEM. p-values: ${ }^{*} p<0.05,{ }^{*} \mathrm{p}<0.01, * * * \mathrm{p}<0.001$; Mann-Whitney test. n.s: non-significant. See also Fig. S8. 

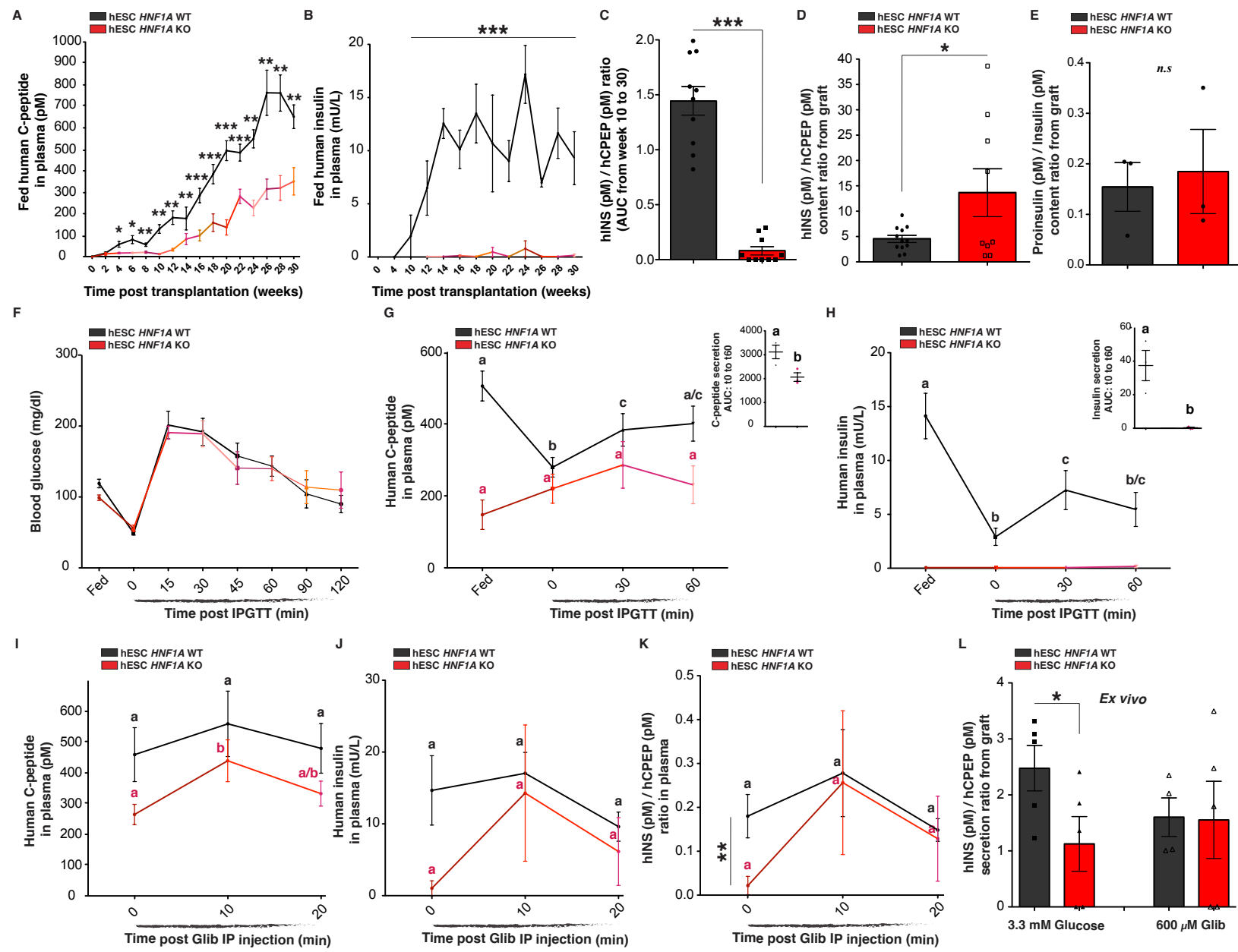

Fig. 4. HNF1A deficiency alters the stoichiometry of insulin to C-peptide secretion in vivo.

(A) Plasma human C-peptide (pM) and (B) human insulin secretion (mU/L) in plasma of ad libitum-fed mice transplanted with hESC-derived endocrine cells ( HNF1A WT n=18 and KO n=7).

(C) hINS(pM)/hCPEP(pM) ratios displayed as area under the curve (AUC) from week 10 to week 30 post-transplantation. (D and E) Content ratio from isolated grafts: (D) hINS(pM)/hCPEP(pM) content ratio and (E) Proinsulin(pM)/Insulin(pM) content ratio. (F-H) IPGTT in mice transplanted with hESC-derived endocrine cells $(H N F 1 A$ WT $\mathrm{n}=10$ and $\mathrm{KO} \mathrm{n}=8)$ in ad libitum-fed state and during an iPGTT (t0, t30 and t60). (F) Blood glucose concentrations (mg/dl), (G) human C-peptide $(\mathrm{pM})$ and $(\mathbf{H})$ human insulin secretion $(\mathrm{mU} / \mathrm{L})$ in plasma. AUC: area under the curve from t0 to t60. p-values were b: $p<0.001, \mathrm{c}: \mathrm{p}<0.05, \mathrm{a} / \mathrm{c}: \mathrm{p}<0.05$ and $\mathrm{b} / \mathrm{c}: \mathrm{p}<0.01$; two-tailed t-test. (I-K) 
Glibenclamide injection in ad libitum-fed mice transplanted with hESC-derived endocrine cells (HNF1A WT $\mathrm{n}=6$ and $\mathrm{KO} \mathrm{n}=5)$. (I) Human C-peptide secretion (pM), (J) human insulin secretion $(\mathrm{mU} / \mathrm{L})$ and $(\mathbf{K})$ hINS $(\mathrm{pM}) / \mathrm{hCPEP}(\mathrm{pM})$ secretion ratios. $\mathrm{p}$-values were b: $\mathrm{p}<0.05$. (L) $\mathrm{hINS}(\mathrm{pM}) / \mathrm{hCPEP}(\mathrm{pM})$ secretion ratios ex vivo in response to indicated secretagogues. All mice were transplanted with clusters of hESC-derived endocrine cells at day 27 of differentiation, and grafts were isolated 30 weeks post-transplantation for ex vivo analysis. All protein concentrations were measured by ELISA. (n) represents the number of biological replicates. For scatter plots, each point in plots represents an independent biological experiment (n). Data are represented as mean \pm SEM. Different letters designate significant differences within group. p-values: ${ }^{*} p<0.05$, ${ }^{* *} \mathrm{p}<0.01,{ }^{* * *} \mathrm{p}<0.001 ;$ two-tailed t-test. n.s: non-significant. See also Fig. S8. 
A

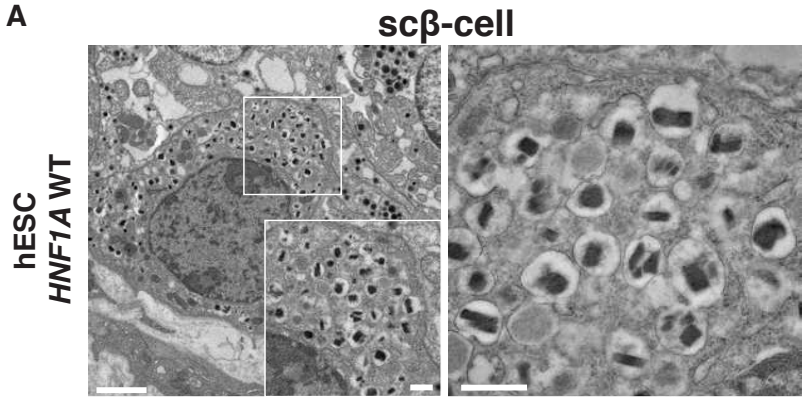

B
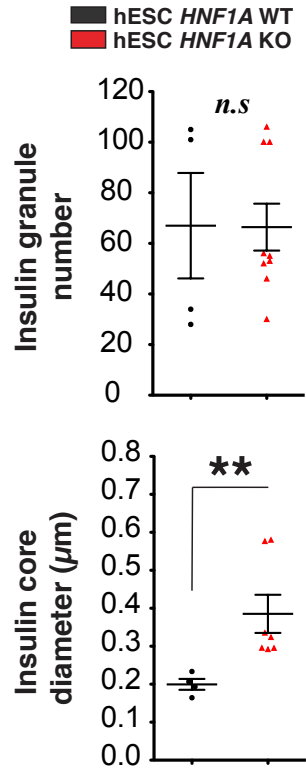

G

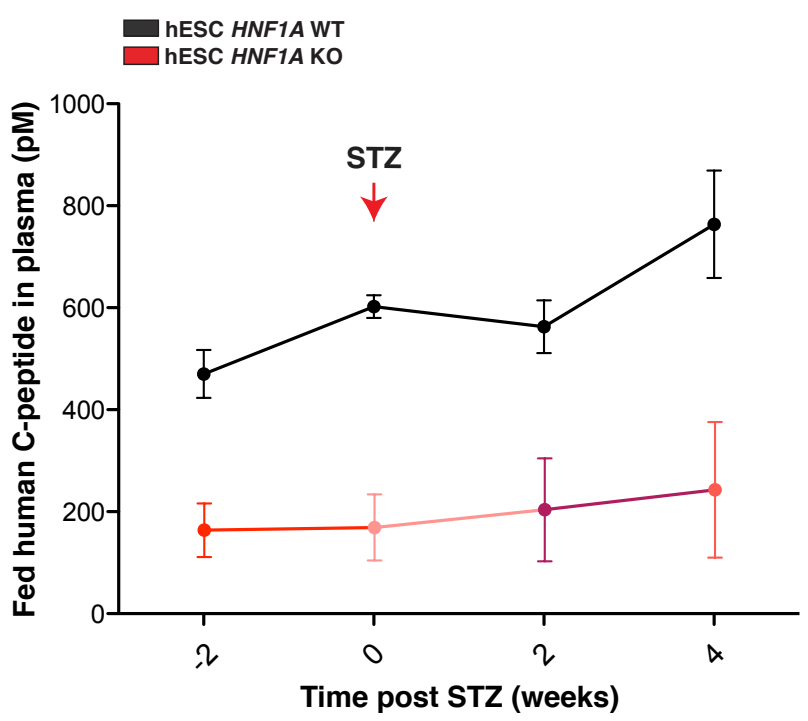

C

F

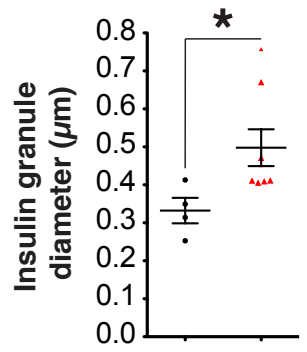

E

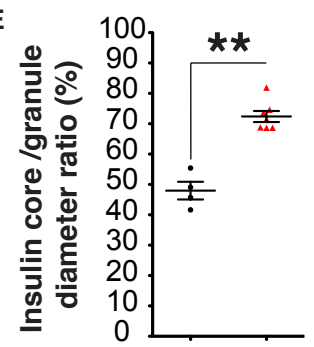

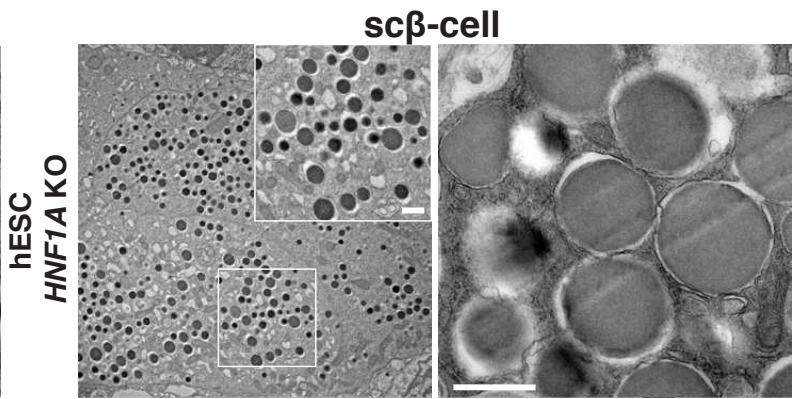

sc $\beta$-cell

岱点
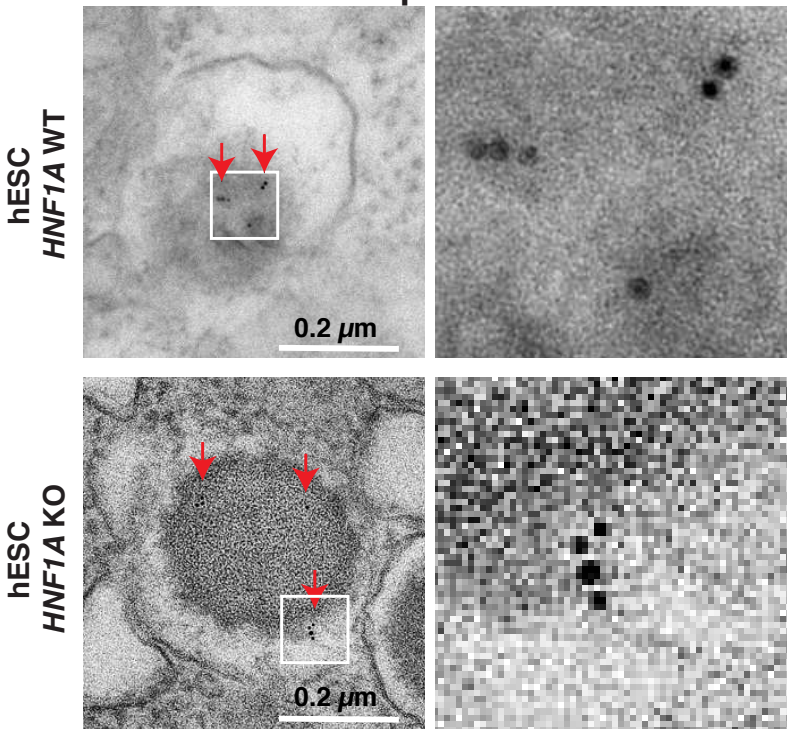

H
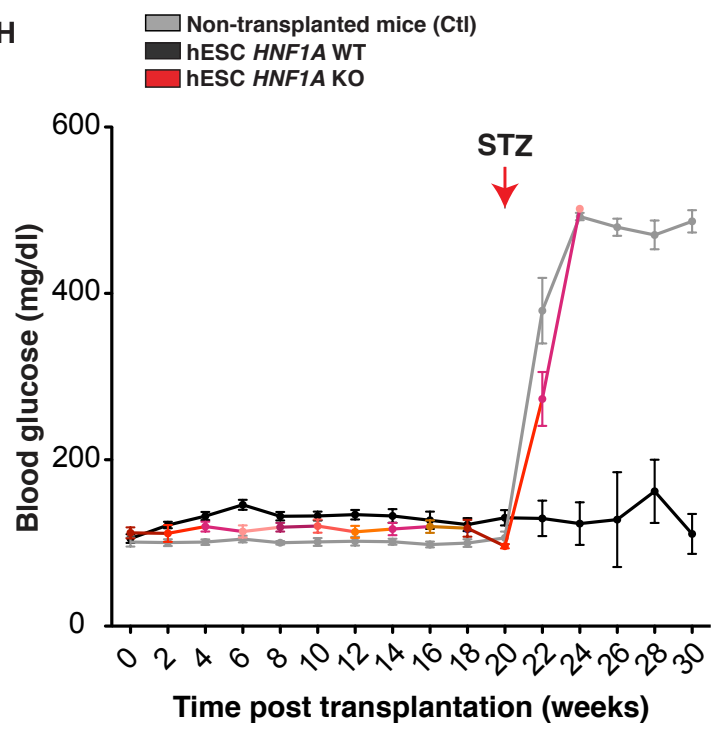
Fig. 5. HNF1A deficiency causes abnormal insulin granule structure and fails to maintain glucose homeostasis in diabetic mice. (A and B) Electron microcopy (EMC) analysis of isolated grafts 30 weeks post-transplantation for hESC-derived endocrine cells (HNF1A WT and KO). Explants are from euglycemic mice. (A) Representative EMC images of sc $\beta$-cells with (B) quantification of insulin granule number, $(\mathbf{C})$ insulin granule diameter $(\mu \mathrm{m})$, (D) insulin granule core diameter $(\mu \mathrm{m})$ and $(\mathbf{E})$ insulin granule core diameter to insulin granule diameter ratio (\%) per cell. Each point in plots is the average of insulin granules per sc $\beta$-like-cells. (F) Representative EMC images of sc $\beta$-cells with human C-peptide immunogold labelling. Scale bars: $2 \mu \mathrm{m}$ in low and $0.5 \mu \mathrm{m}$ in high magnification. (G) Fed human C-peptide (pM) in plasma of ad libitum-fed mice transplanted with hESC-derived endocrine cells (HNF1A WT n=8 and KO n=3) measured weeks before (-2) and after STZ treatment (0, 2 and 4). (H) Blood glucose concentrations (mg/dl) in ad libitum-fed mice transplanted with hESC-derived endocrine cells (HNF1A WT $\mathrm{n}=18$ and KO $\mathrm{n}=10)$ or without cells $(\mathrm{Ctl} \mathrm{n}=14)$. Mice $(H N F 1 A$ WT $\mathrm{n}=6, \mathrm{KO} \mathrm{n}=3$ and $\mathrm{Ctl} \mathrm{n}=5)$ were injected (red arrow) with streptozotocin (STZ). All mice were transplanted with hESC-derived endocrine cells at day 27 of differentiation, and grafts were isolated 30 weeks post-transplantation for ex vivo analysis. All protein concentrations were measured by ELISA. (n) represents the number of biological replicates. For scatter plots, each point in plots represents an independent biological experiment (n). Data are represented as mean \pm SEM. p-values: ${ }^{*} p<0.05, * * p<0.01, * * * p<0.001$; Mann-Whitney test. n.s: non-significant. See also Fig. S9 and S10. 

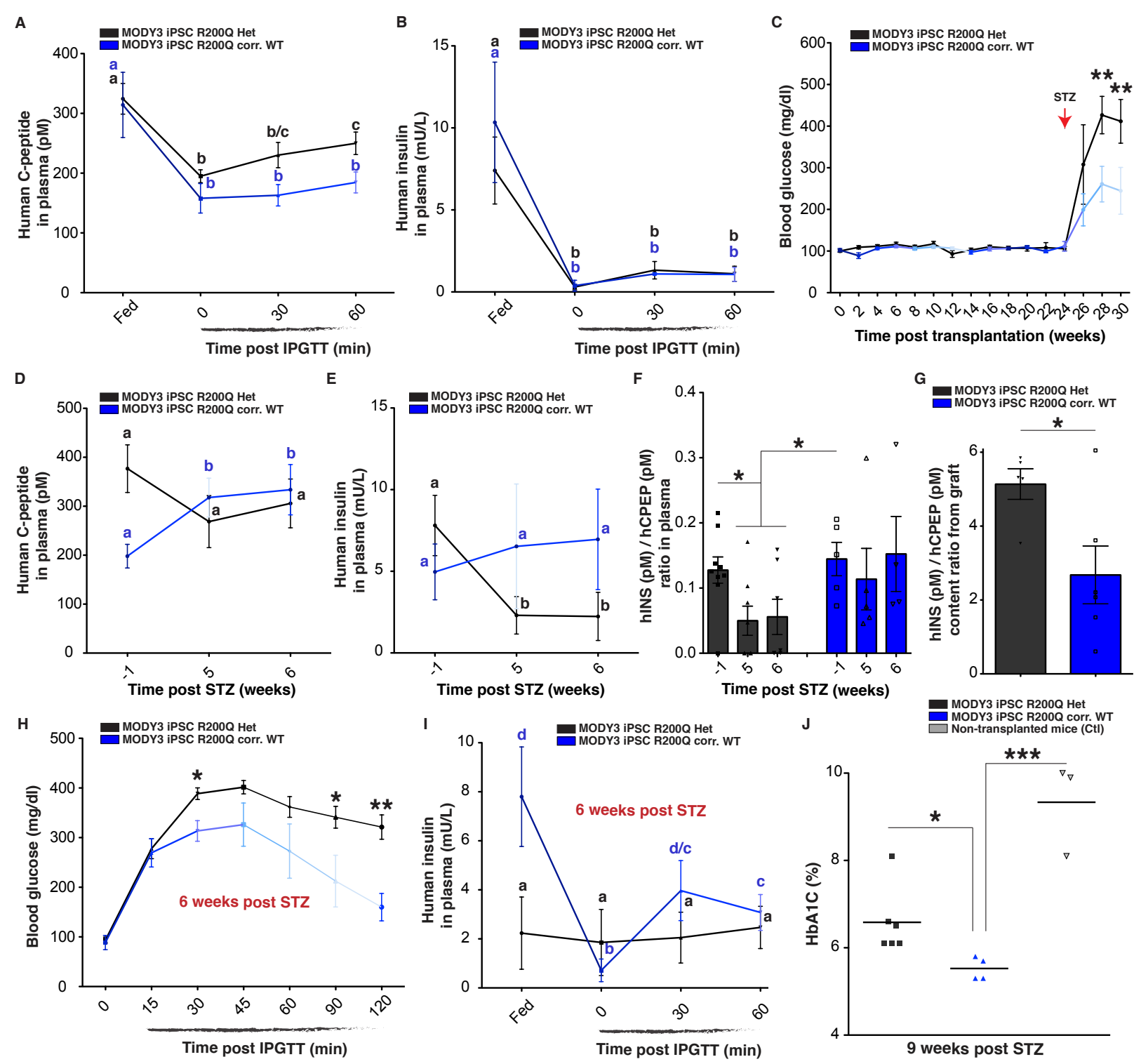

G

MODY3 iPSC R200Q Het
MODY3 iPSC R200Q corr. WT

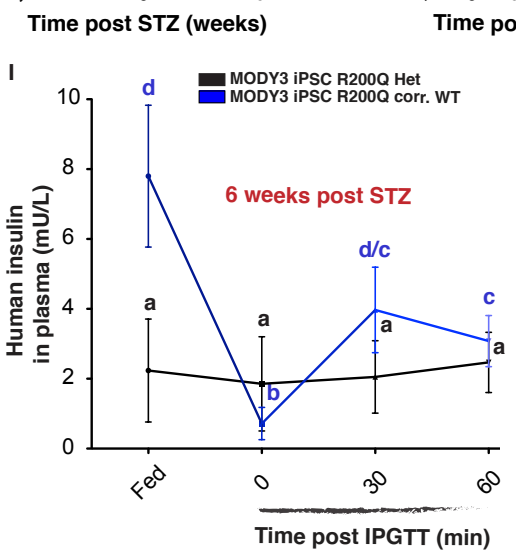

$J$

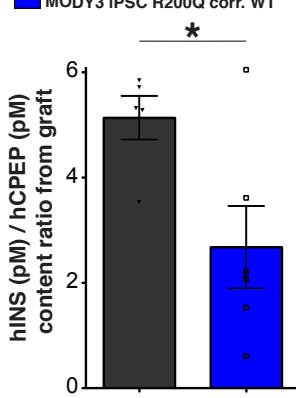

MODY3 3 PSC R200Q Het MODY3 iPSC R200Q corr. WT (CtI)

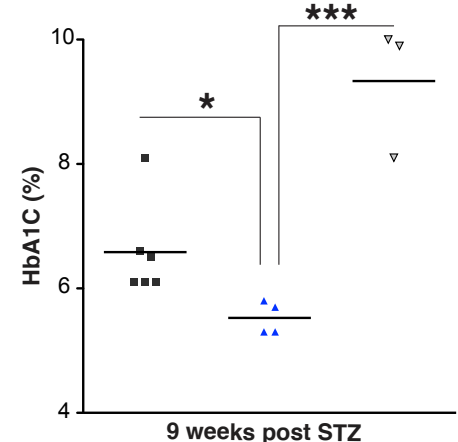

Fig. 6. HNF1A haploinsufficiency gradually impairs sc $\beta$-cell function in vivo. (A-C) Human C-peptide (pM) and human insulin levels in plasma of ad libitum-fed mice transplanted with MODY3 iPSC-derived endocrine cells (HNF1A+/R200Q and R200Q-corrected WT). (A) Plasma human C-peptide (pM) and (B) human insulin secretion (mU/L) in mice transplanted with MODY3 iPSC-derived endocrine cells (R200Q Het n=10 and R200Q corr. WT n=5) in ad libitum-fed state and during an iPGTT (t0, t30 and t60). p-values were b: $p<0.001, b / c: p<0.01$ and $c: p<0.05$. (C) Blood glucose concentrations $(\mathrm{mg} / \mathrm{dl})$ in ad libitum-fed mice transplanted with MODY3 iPSC- 
derived endocrine cells (R200Q Het $n=21$ and R200Q corr. WT $n=32$ ). Mice (R200Q Het $n=8$, R200Q corr. WT $n=8$ and Ctl n=5) were injected (red arrow) with STZ. (D) Human C-peptide (pM), (E) human insulin (mU/L) (R200Q Het $\mathrm{n}=9$ and R200Q corr. WT $\mathrm{n}=6$ ), and (F) hINS $(\mathrm{pM}) / \mathrm{hCPEP}(\mathrm{pM})$ secretion ratios monitored in plasma of ad libitum-fed mice weeks before (-1) and after (5 and 6) STZ treatment. $p$-values were b and d: $p<0.01 ; c: p<0.05$. (G) Quantification of hINS(pM)/hCPEP(pM) content ratios from isolated grafts. (H-I) IPGTT in mice transplanted with MODY3 iPSC-derived endocrine cells (R200Q Het n=5 and R200Q corr. WT n=4) 6 weeks post STZ treatment in ad libitum-fed state and during an iPGTT (t0, t30 and t60). (H) Blood glucose concentrations ( $\mathrm{mg} / \mathrm{dl})$ and $(\mathbf{I})$ human insulin secretion $(\mathrm{mU} / \mathrm{L})$ in plasma. p-values were $\mathrm{b} p<0.01, \mathrm{~d} / \mathrm{c}$ and $\mathrm{c}: \mathrm{p}<0.05$. (R200Q Het $\mathrm{n}=5$ and R200Q corr. WT $\mathrm{n}=4$ ). (J) HbA1C (\%) in mice transplanted with MODY3 -iPSC-derived endocrine cells or non-transplanted (Ctl) 9 weeks after STZ treatment. All mice were transplanted with iPSC-derived endocrine cells at day 27 of differentiation, and grafts were isolated 30-35 weeks post-transplantation for ex vivo analysis. All protein concentrations were measured by ELISA. For scatter plots, each point in plots represents an independent biological experiment (n). Data are represented as mean \pm SEM. Different letters designate significant differences within groups. p-values were ${ }^{*} \mathrm{p}<0.05,{ }^{*} \mathrm{p}<0.01,{ }^{* *} \mathrm{p}<0.001$ two-tailed t-test. n.s: non-significant. See also Fig. S11-S13. 


\section{Supplementary Files}

This is a list of supplementary files associated with this preprint. Click to download.

- SupplementalTables.xlsx

- SupplementalMaterial5.4.21compressed.pdf 